\title{
POLARIZATION MODES IN A STRONGLY MAGNETIZED HYDROGEN GAS
}

\author{
Tomasz Bulik \\ University of Chicago, Astr.\& Astrophysics and Enrico Fermi Institute, 5640 S. Ellis Av., \\ Chicago, IL 60615 \\ and \\ George G. Pavlov 円 \\ Pennsylvania State University, 525 Davey Lab., University Park, PA 16802 \\ E-mail: tomek@gamma.uchicago.edu,pavlov@astro.psu.edu
}

\begin{abstract}
Propagation of high-frequency radiation in an anisotropic medium can be described in terms of two normal modes with different polarizations and different absorption coefficients. We investigate the properties of the normal modes in a strongly magnetized hydrogen gas for conditions expected in atmospheres of isolated neutron stars. We use the Kramers-Kronig relations to obtain the polarizability tensor for the strongly magnetized hydrogen. We derive and compute the polarizations and absorption coefficients of the normal modes from the polarizability tensor using both analytical approximations and numerical calculations. We find that the spectral features and anisotropy associated with the bound-bound and bound-free transitions in the magnetized hydrogen are manifested in the polarization characteristics, which affects substantially the spectral and angular dependences of the absorption coefficients of the normal modes and the transfer of radiation in neutron star atmospheres. There exist at least two critical frequencies where, for any direction of propagation, either the normal mode polarizations are exactly linear or orientations of two polarization ellipses coincide with each other. For each of these frequencies, there exist a direction of propagation for which the two normal modes are linearly polarized, and their polarizations and absorption coefficients fully coincide. The unusual properties of the normal modes should manifest themselves in the spectra, angular distribution, and polarization of the thermal-like radiation emitted from surface layers of neutron stars.
\end{abstract}

\footnotetext{
${ }^{1}$ On leave from A. F. Ioffe Physico-Technical Institute, St. Petersburg, 194021, Russia
} 
Subject headings: atomic processes - magnetic fields - pulsars: general - stars: atmospheres - stars: neutron

\section{INTRODUCTION}

With the development of observational capabilities of space X-ray/UV observatories and ground based telescopes it is now feasible to detect radiation from surface layers of neutron stars with rather low temperatures, $T \sim(0.2-10) \times 10^{5} \mathrm{~K}$ (Ögelman 1995; Pavlov, Stringfellow \& Córdova 1995). The immense magnetic fields of neutron stars, $B \sim 10^{11}-10^{13} \mathrm{G}$, substantially increase the ionization potentials of atoms (e. g., Canuto \& Ventura 1977): $I \sim Z^{2} \operatorname{Ry} \ln ^{2}\left(\gamma / Z^{2}\right)$ at $\gamma \gg Z^{2}$, where $\gamma=\hbar \omega_{B e} /(2 \mathrm{Ry})=B /\left(2.35 \times 10^{9}\right.$ $\mathrm{G})$ is the magnetic field in the atomic units, $\omega_{B e}=e B / m_{e} c$ is the electron cyclotron frequency, $Z$ is the effective ion charge, and $\mathrm{Ry}=m_{e} e^{4} / 2 \hbar^{2}=13.6 \mathrm{eV}$ is the Rydberg energy. This means that even light atoms may be only partly ionized (or even fully nonionized) in neutron star atmospheres, and be the main contributors to the atmosphere opacity (Pavlov et al. 1995a). Thus, to model the atmospheres for further interpretation of the observational results, it is necessary to investigate the effect of the strongly magnetized atoms on absorption and propagation of radiation.

It is well known (see, e.g., Gnedin \& Pavlov 1974) that radiation propagates in an anisotropic medium in form of two so-called normal modes (NMs) which have different polarizations and, consequently, different absorption and refraction coefficients. The dependence of the NM absorption coefficients on the frequency and direction of propagation determines the spectrum and angular distribution of radiation emitted from an anisotropic medium. Properties of the NMs have been thoroughly investigated (e.g., Pavlov, Shibanov

\& Yakovlev 1980; Kaminker, Pavlov \& Shibanov 1982, 1983; Mészáros 1992, and references therein) for the case of fully ionized plasma, when polarization and absorption of the modes are determined by the free-free transitions and Thomson scattering in the magnetic field. However, there has been no analysis of how these properties would change if the bound-bound and bound-free transitions in the strongly magnetized atoms are important.

To find the polarizations of the normal modes, one should proceed from the polarizability tensor of the anisotropic medium. The contribution of the bound-bound and bound-free transitions to the components of the tensor is determined by the structure of the strongly magnetized atoms. For the simplest hydrogen atom, assuming the center of 
mass of the atom is fixed, the atomic structure and probabilities of the radiative transitions, which determine the antihermitian part of the polarizability tensor, have been investigated in detail (e. g., Canuto \& Ventura 1977, Forster et al. 1984, Potekhin \& Pavlov 1993). The hermitian part of the polarizability has not been discussed, to the best of our knowledge, even under this oversimplified assumption. Moreover, it has been understood recently that motion of atoms in strong magnetic fields changes qualitatively their structure (Herold et al. 1981; Vincke \& Baye 1988; Vincke, Le Dourneuf \& Baye 1992; Pavlov \& Mészáros 1993; Potekhin 1994). This occurs because the motion across the magnetic field induces an electric field, $\mathbf{F}=c^{-1} \mathbf{V} \times \mathbf{B}$, which breaks the cylindrical symmetry and couples the center-of-mass motion to the internal structure of the atom. The energies and wave functions of the moving atom depend on the transverse component $\mathbf{K}_{\perp}$ of a generalized momentum $\mathbf{K}$. When $\mathbf{K}_{\perp}$ is small (at low temperatures of the gas), the perturbation approach is applicable, and the coupling effect can be described in terms of an effective transverse mass of the atom (Vincke \& Baye 1988; Pavlov \& Mészáros 1993). This transverse mass always exceeds the mass of the atom without the magnetic field. It grows with the magnetic field, being and is generally higher for more excited atomic states. When $\mathbf{K}_{\perp}$ increases beyond the applicability of the perturbation theory, the induced electric field causes decentering of the atom, i. e., the electron gets into a potential well which is shifted from the proton more for the larger $\mathbf{K}_{\perp}$. These decentered atoms have very unusual properties. For instance, their velocity decreases with increasing $\mathbf{K}_{\perp}$ because the growth of $\mathbf{K}_{\perp}$ goes not to acceleration of the atom but to larger decentering. The decentered states have relatively small binding energies, so that they can be occupied only at relatively high temperatures and not too high densities, when the size of the atom does not exceed the mean distance between the particles.

Qualitative effects of the coupling on the radiative transitions were discussed by Pavlov \& Mészáros (1993). They show, in particular, that different dependence of different atomic levels on $\mathbf{K}_{\perp}$ leads to a new broadening mechanism of the spectral lines and photoionization edges. The "magnetic coupling width" exceeds the Doppler width by orders of magnitude, growing proportional to the gas temperature. More detailed treatment of the radiative transitions, with allowance for the decentering of moving atoms, has been presented by Bezchastnov \& Potekhin (1994) for bound-free transitions and by Pavlov \& Potekhin (1995) for bound-bound transitions.

In the present paper we will consider the polarizability tensor and properties of the polarization modes of the hydrogen gas for the case of relatively low temperatures, when interaction of the radiation with the medium is mainly determined by the nonionized atoms. At these low temperatures, most of the atoms occupy the ground level, and, at densities typical for neutron star atmospheres, one can neglect contribution of the decentered states. 
In these conditions, the coupling effects can be considered in the frame of the perturbation approach. In Section 2 we recall general equations for the polarizability tensor, normal mode polarizations and absorption coefficients, and describe our approach for calculation of these quantities. We present numerical results and analytical limits in Section 3. In Section 4 we discuss some consequences of the obtained results for neutron star atmosphere modeling.

\section{BASIC EQUATIONS}

\subsection{Polarization Characteristics of the Normal Modes.}

The propagation of electromagnetic waves in a magnetized medium is fully described by the dielectric tensor

$$
\epsilon_{i k}=\delta_{i k}+4 \pi \chi_{i k}
$$

where $\chi_{i k}$ is the polarizability tensor. In the case of a tenuous medium, (i. e., at $\left|n_{j}-1\right|<1$, where $j=1,2$ labels the NM, and $n_{j}$ is the complex refraction index) the NMs are transverse, and the (real) refraction index $\kappa_{j}$ and the absorption coefficient $\mu_{j}$ are given by (Pavlov et al. 1980)

$$
n_{j}=\kappa_{j}+i \frac{c}{2 \omega} \mu_{j}=n_{I} \pm \sqrt{n_{L}^{2}+n_{C}^{2}}
$$

where

$$
\begin{gathered}
n_{I}=1+\pi\left(\chi_{y y}+\chi_{x x} \cos ^{2} \theta-\chi_{x z} \sin 2 \theta+\chi_{z z} \sin ^{2} \theta\right) \\
n_{L}=\pi\left(\chi_{y y}-\chi_{x x} \cos ^{2} \theta+\chi_{x z} \sin 2 \theta-\chi_{z z} \sin ^{2} \theta\right) \\
n_{C}=2 \pi i\left(\chi_{x y} \cos \theta+\chi_{y z} \sin \theta\right)
\end{gathered}
$$

Here $\theta$ is the angle between the wave vector $\mathbf{q}$ and the magnetic field $\mathbf{B}$, and $\chi_{i k}$ is the polarizability tensor in the coordinate frame with the $z$-axis along $\mathbf{B}$ and the $x$-axis in the B-q plane. The NMs can be described by the ellipticity $\mathcal{P}_{j}$ (the modulus $\left|\mathcal{P}_{j}\right|$ is the ratio of the minor axis to the major axis of the polarization ellipse; the sign of $\mathcal{P}_{j}$ determines the direction of rotation of the polarization vector) and the position angle $\delta_{j}$ between the major axis of the polarization ellipse and the projection of $\mathbf{B}$ onto the plane perpendicular to q. The ellipticity and position angle are determined by the modulus $r_{j}$ and phase $\varphi_{j}$ of the complex quantity

$$
r_{j} \exp \left(i \varphi_{j}\right)=\frac{-n_{C} \pm \sqrt{n_{L}^{2}+n_{C}^{2}}}{n_{L}}
$$




$$
\mathcal{P}_{j}=\frac{r_{j}-1}{r_{j}+1}, \quad \delta_{j}=\frac{\varphi_{j}}{2} .
$$

It follows from these equations that the NM properties can be conveniently analyzed with the use of real parameters $q$ and $p$ defined as (Gnedin \& Pavlov 1974)

$$
q+i p=\frac{n_{L}}{n_{C}}
$$

The NM polarizations are nearly orthogonal $\left(\delta_{2} \simeq \delta_{1} \pm \pi / 2 ; \delta_{j} \simeq 0\right.$ or $\pi / 2 ;$ and $\left.\mathcal{P}_{2}=-\mathcal{P}_{1}\right)$ when $2|p| \ll p^{2}+q^{2}+1$. In particular, they are orthogonal when $p^{2} \ll q^{2}$ (the most common case, which is usually realized in a wide range of frequencies and angles), or when $p^{2} \gg q^{2}$ and $|p| \gg 1$. In the case when $p^{2} \ll q^{2}$, the (orthogonal) NMs are linearly polarized, $\left|\mathcal{P}_{1}\right|=\left|\mathcal{P}_{2}\right| \simeq 0$, if $|q| \gg 1$, and they are circularly polarized, $\left|\mathcal{P}_{1}\right|=\left|\mathcal{P}_{2}\right| \simeq 1$, if $|q| \ll 1$. If $p^{2} \gg q^{2}$ and $q^{2} \ll 1$, then the NMs are linearly polarized at $|p| \geq 1$, and the polarization ellipses coincide $\left[\delta_{1} \simeq \delta_{2} \simeq(\pi / 4) \operatorname{sign}(p), \mathcal{P}_{1}=-\mathcal{P}_{2}\right]$ at $|p| \leq 1$. In the special case when $q=0$ and $|p|=1$, the NM polarizations are purely linear and coincide with each other (are "completely non-orthogonal"): $\mathcal{P}_{1}=\mathcal{P}_{2}=0, \delta_{1}=\delta_{2}=(\pi / 4) \operatorname{sign}(p)$. For further details see, e. g., Pavlov et al. (1980).

\subsection{General Equations for the Polarizability Tensor in the Dipole Approximation.}

In the dipole approximation, which is justified for frequencies that are not too high (see, e. g., Pavlov \& Potekhin 1995 for a discussion), the polarizability tensor of a magnetized gas is diagonal in the cyclic coordinates, $\chi_{\alpha \beta}=\chi_{\alpha} \delta_{\alpha \beta}$, where $\alpha, \beta=0, \pm 1$, and the cyclic unit vectors are defined as $\mathbf{e}_{0}=e_{z}, \mathbf{e}_{ \pm 1}=2^{-1 / 2}\left(\mathbf{e}_{x} \pm i \mathbf{e}_{\mathbf{y}}\right)$. In terms of the cyclic components $\chi_{\alpha}$, we have

$$
\begin{gathered}
n_{I}=1+\pi\left[\left(\chi_{+1}+\chi_{-1}\right) \frac{1+\cos ^{2} \theta}{2}+\chi_{0} \sin ^{2} \theta\right], \\
n_{L}=\pi\left(\frac{\chi_{+1}+\chi_{-1}}{2}-\chi_{0}\right) \sin ^{2} \theta \\
n_{C}=\pi\left(\chi_{-1}-\chi_{+1}\right) \cos \theta
\end{gathered}
$$

and

$$
q+i p=(\tilde{q}+i \tilde{p}) \frac{\sin ^{2} \theta}{2 \cos \theta}, \quad \tilde{q}+i \tilde{p}=\frac{\chi_{+1}+\chi_{-1}-2 \chi_{0}}{\chi_{-1}-\chi_{+1}}
$$


The polarizability tensor is the sum of the hermitian and antihermitian parts, $\chi_{\alpha}=\chi_{\alpha}^{H}+i \chi_{\alpha}^{A}$, responsible for refraction and absorption of radiation. The antihermitian part can be written (for a single component ideal gas) as

$$
\begin{aligned}
\chi_{\alpha}^{A} & =\frac{\omega_{p a}^{2}}{8 \omega} \sum_{i f} \xi_{i}\left[f_{i f}^{\alpha} \delta\left(\omega-\omega_{f i}\right)-f_{f i}^{\alpha} \delta\left(\omega-\omega_{i f}\right)\right] \\
& =\frac{\omega_{p a}^{2}}{8 \omega} \sum_{i f}^{\prime}\left(\xi_{i}-\xi_{f}\right)\left[f_{i f}^{\alpha} \delta\left(\omega-\omega_{f i}\right)-f_{f i}^{\alpha} \delta\left(\omega-\omega_{i f}\right)\right],
\end{aligned}
$$

where $\omega_{p a}=\left(4 \pi n_{a} e^{2} / m_{e}\right)^{1 / 2}$ is the "plasma frequency" of bound electrons, $n_{a}$ is the number density of atoms, $\xi_{i}=n_{a i} / n_{a}$ is the population of the quantum state $i, \omega_{f i}=\left(E_{f}-E_{i}\right) / \hbar$ is the transition frequency, and $f_{i f}$ is the oscillator strength for the transition $i \rightarrow f$,

$$
f_{i f}^{\alpha}=\frac{2 m_{e} \omega_{f i}}{\hbar}\left|\left(\mathbf{r}_{f i} \cdot \mathbf{e}_{\alpha}\right)\right|^{2}=\frac{2 m_{e} \omega_{f i}}{\hbar}\left|\left(x_{-\alpha}\right)_{f i}\right|^{2} .
$$

The double sum $\sum_{i f}$ implies summation (and integration over the continuum part of the energy spectrum) over all possible combinations of $i$ and $f$ (except $i=f$ ); in the sum $\sum_{i f}^{\prime}$ only the states are summed (integrated) for which $E_{f}>E_{i}$. The general equation for the hermitian part can be written, in the same approximation, as

$$
\chi_{\alpha}^{H}=-\frac{\omega_{p a}^{2}}{8 \pi \omega} \sum_{i f} \xi_{i}\left[\frac{f_{i f}^{\alpha}}{\omega-\omega_{f i}}-\frac{f_{f i}^{\alpha}}{\omega-\omega_{i f}}\right]=-\frac{\omega_{p a}^{2}}{8 \pi \omega} \sum_{i f}^{\prime}\left(\xi_{i}-\xi_{f}\right)\left[\frac{f_{i f}^{\alpha}}{\omega-\omega_{f i}}-\frac{f_{f i}^{\alpha}}{\omega-\omega_{i f}}\right] .
$$

It follows from the completeness of the wave functions that

$$
\sum_{f} \frac{1}{\omega_{f i}}\left(f_{i f}^{\alpha}+f_{f i}^{\alpha}\right)=0
$$

which means, in particular, that $\chi_{\alpha}^{H}$ has no pole at $\omega=0$ if all the electrons are bound.

We see from these equations that direct calculation of $\chi^{H}$ implies an additional integration over the continuum states, as compared to $\chi^{A}$, because there are no deltafunctions in equation (16), and this integration may not be straightforward. An alternative way to find the hermitian part is to use the Kramers-Kronig (KK) relation (Guilbert transformation of the antihermitian part):

$$
\chi_{\alpha}^{H}(\omega)=\frac{\mathcal{P}}{\pi} \int_{-\infty}^{\infty} \frac{\chi_{\alpha}^{A}\left(\omega^{\prime}\right)}{\omega^{\prime}-\omega} \mathrm{d} \omega^{\prime} \equiv \hat{K}\left[\chi_{\alpha}^{A}(\omega)\right]
$$

which is derived from the general analytical properties of the polarizability: $\chi(\omega)$ is a regular function in the upper half-plane of complex $\omega ; \chi(\omega)$ has no singularities on the 
real axis except possibly a simple pole at the origin; $\chi(\omega \rightarrow \infty) \rightarrow 0$ (see e.g. Landau \& Lifshitz, 1960). Since the quantity $\omega \chi_{\alpha}(\omega)$ also has all the necessary analytical properties, we may apply the KK transformation to this product

$$
\chi_{\alpha}^{H}(\omega)=\frac{1}{\omega} \hat{K}\left[\omega \chi_{\alpha}^{A}(\omega)\right]=\frac{c}{4 \pi \omega} \hat{K}\left[\mu_{\alpha}(\omega) \Theta(\omega)+\mu_{-\alpha}(-\omega) \Theta(-\omega)\right],
$$

where $\Theta(x)=1$ for $x \geq 0, \Theta(x)=0$ for $x<0, \mu_{\alpha}(\omega)$ is the absorption coefficient (defined for $\omega>0)$ :

$$
\mu_{\alpha}(\omega)=\frac{\pi \omega_{p a}^{2}}{2 c} \sum_{i f}^{\prime}\left(\xi_{i}-\xi_{f}\right) f_{i f}^{\alpha} \delta\left(\omega-\omega_{f i}\right)=n_{a}[1-\exp (-\hbar \omega / k T)] \sum_{i} \xi_{i} \sigma_{i}(\omega),
$$

where $\sigma_{i}(\omega)$ is the absorption cross section, and $T$ is the temperature. To derive equation (19), we used the property of the oscillator strength

$$
f_{i f}^{\alpha}=-f_{f i}^{-\alpha}
$$

which implies the symmetry relations

$$
\chi_{\alpha}^{A}(-\omega)=-\chi_{-\alpha}^{A}(\omega), \quad \chi_{\alpha}^{H}(-\omega)=\chi_{-\alpha}^{H}(\omega) .
$$

Applying the KK transformation to $\omega \chi_{\alpha}$ instead of $\chi_{\alpha}$ is technically easier because the

additional $\omega$ cancels the false pole (for bound electrons) of $\chi_{\alpha}$ at $\omega=0$. Thus, to find $\chi^{H}$ in this way, one needs the absorption coefficients at $\omega>0$ for the longitudinal $(\alpha=0)$, right $(\alpha=+1)$, and left $(\alpha=-1)$ polarizations.

\subsection{Oscillator Strengths and Cross Sections for a Strongly Magnetized Hydrogen Atom at Rest}

The absorption coefficients for the bound atoms are contributed from the bound-bound and bound-free transitions. For isolated atoms at rest, the problem of calculating the bound-bound absorption coefficients is reduced to calculating the oscillator strengths $f_{f i}^{\alpha}$. When the magnetic field $\mathbf{B}=B \mathbf{e}_{z}$ is so strong that the ratio of the electron cyclotron energy, $\hbar \omega_{B e}=2 \gamma \mathrm{Ry}=11.6 B_{12} \mathrm{keV}$, substantially exceeds the binding energy, $\sim$ Ry $\ln ^{2} \gamma$ at $\ln ^{2} \gamma \gg 1$, the adiabatic approximation is valid. In this approximation, the bound states of the hydrogen atom are described by the Landau number $N=0,1,2, \ldots$, the negative of the $z$-projection of the angular momentum $s=-N,-N+1,-N+2, \ldots$, and the longitudinal quantum number $n=0,1,2, \ldots$ (see, e. g., Canuto and Ventura 1977 for details). The wave 
functions are the products of the Landau states $\Phi_{N s}\left(\mathbf{r}_{\perp}\right)$ and longitudinal wave functions $g_{N s n}(z)$ which obey a one-dimensional Schrödinger equation. The oscillator strengths of the transitions between the states $\kappa=(N s n)$ and $\kappa^{\prime}=\left(N^{\prime} s^{\prime} n^{\prime}\right)$ are given by the following equations

$$
\begin{aligned}
f_{\kappa \kappa^{\prime}}^{-1} & =\frac{2 \omega_{\kappa^{\prime} \kappa}}{\omega_{B e}}\left[(N+s) \delta_{N^{\prime} N}+(N+1) \delta_{N^{\prime}, N+1}\right]\left|Z_{\kappa^{\prime} \kappa}^{(0)}\right|^{2} \delta_{s^{\prime}, s-1} \\
f_{\kappa \kappa^{\prime}}^{+1} & =\frac{2 \omega_{\kappa^{\prime} \kappa}}{\omega_{B e}}\left[(N+s+1) \delta_{N^{\prime} N}+N \delta_{N^{\prime}, N-1}\right]\left|Z_{\kappa^{\prime} \kappa}^{(0)}\right|^{2} \delta_{s^{\prime}, s+1}, \\
f_{\kappa \kappa^{\prime}}^{0} & =\frac{\hbar \omega_{\kappa^{\prime} \kappa}}{\mathrm{Ry}} \delta_{s^{\prime} s} \delta_{N^{\prime} N}\left|Z_{\kappa^{\prime} \kappa}^{(1)}\right|^{2},
\end{aligned}
$$

where

$$
Z_{\kappa^{\prime} \kappa}^{(0)}=\int_{-\infty}^{\infty} \mathrm{d} z g_{\kappa^{\prime}}^{*}(z) g_{\kappa}(z) \quad \text { and } \quad Z_{\kappa^{\prime} \kappa}^{(1)}=\int_{-\infty}^{\infty} \mathrm{d} z g_{\kappa^{\prime}}^{*}(z)\left(z / a_{B}\right) g_{\kappa}(z)
$$

are the overlapping integral and dimensionless matrix element of the longitudinal coordinate, respectively; $a_{B}$ is the Bohr radius. The transition frequency is

$$
\omega_{\kappa^{\prime} \kappa}=\left(N^{\prime}-N\right) \omega_{B e}+\left(N^{\prime}-N+s^{\prime}-s\right) \omega_{B p}+\tilde{\omega}_{\kappa^{\prime} \kappa}
$$

where $\hbar \tilde{\omega}_{\kappa^{\prime} \kappa}=\epsilon_{\kappa^{\prime}}-\epsilon_{\kappa}$ is the difference of the longitudinal energies, and $\omega_{B p}=\left(m_{e} / m_{p}\right) \omega_{B e}$ is the proton cyclotron frequency. In the application to the neutron star atmospheres, the only important case is when the initial state $\kappa$ belongs to the ground Landau level $N=0$ (the excited Landau levels are unpopulated at $k T \ll \hbar \omega_{B e}$ ). Moreover, we will consider temperatures low enough that the population of even the first excited level (010) is small compared to the ground state, (000), which allows us to neglect transitions between the excited levels. Then, putting $\kappa=(000)$, we obtain

$$
\begin{aligned}
f_{\kappa \kappa^{\prime}}^{-1} & =2 \frac{\omega_{B e}+\tilde{\omega}_{1-1 n^{\prime}, 000}}{\omega_{B e}}\left|Z_{1-1 n^{\prime}, 000}^{(0)}\right|^{2} \delta_{N^{\prime} 1} \delta_{s^{\prime}-1}, \\
f_{\kappa \kappa^{\prime}}^{+1} & =2 \frac{\tilde{\omega}_{01 n^{\prime}, 000}+\omega_{B p}}{\omega_{B e}}\left|Z_{01 n^{\prime}, 000}^{(0)}\right|^{2} \delta_{N^{\prime} 0} \delta_{s^{\prime} 1}, \\
f_{\kappa \kappa^{\prime}}^{0} & =\frac{\hbar \tilde{\omega}_{00 n^{\prime}, 000}}{\mathrm{Ry}}\left|Z_{00 n^{\prime}, 000}^{(1)}\right|^{2} \delta_{N^{\prime} 0} \delta_{s^{\prime} 0} .
\end{aligned}
$$

Note that the longitudinal energies and wave functions of the states $(01 n)$ and $(1-1 n)$ coincide (Hasegawa \& Howard 1961). This means that $\tilde{\omega}_{1-1 n^{\prime}, 000}=\tilde{\omega}_{01 n^{\prime}, 000}$ and $Z_{1-1 n^{\prime}, 000}^{(0)}=Z_{01 n^{\prime}, 000}^{(0)}$. Since the parity of the longitudinal states is $(-1)^{n}$, transitions from the ground (even) state are allowed to odd states, $n=1,3, \ldots$, for the longitudinal polarization $\alpha=0$, and to even states, $n=0,2, \ldots$, for the circular polarizations $\alpha= \pm 1$. Moreover, in the strong magnetic field, the overlapping integrals $Z_{01 n^{\prime}, 000}^{(0)}$ for $n^{\prime} \neq 0$ are 
much smaller than that for $n^{\prime}=0$ and can be neglected. As a result, equations (28) - (30) take the form

$$
\begin{aligned}
f_{\kappa \kappa^{\prime}}^{-1} & =2\left(1+\frac{\tilde{\omega}_{010,000}}{\omega_{B e}}\right) \delta_{N^{\prime} 1} \delta_{s^{\prime}-1} \delta_{n^{\prime} 0}, \\
f_{\kappa \kappa^{\prime}}^{+1} & =2\left(\frac{\tilde{\omega}_{010,000}}{\omega_{B e}}+\frac{m_{e}}{m_{p}}\right) \delta_{N^{\prime} 0} \delta_{s^{\prime} 1} \delta_{n^{\prime} 0}, \\
f_{\kappa \kappa^{\prime}}^{0} & =\frac{\hbar \tilde{\omega}_{00 n^{\prime}, 000}}{\mathrm{Ry}}\left|Z_{00 n^{\prime}, 000}^{(1)}\right|^{2} \delta_{N^{\prime} 0} \delta_{s^{\prime} 0} ; \quad n^{\prime}=1,3, \ldots
\end{aligned}
$$

These equations demonstrate that the bound-bound transitions are quite different for different polarizations. The strongest transition (with $f_{\kappa \kappa^{\prime}}^{-} \simeq 2, \omega_{\kappa^{\prime} \kappa} \simeq \omega_{B e}$ ) occurs onto the excited Landau level, $(000) \rightarrow(1-10)$, and it is allowed only for the left polarization. If the electrons were not bound to protons by the Coulomb forces, $\tilde{\omega}_{\kappa^{\prime} \kappa} \rightarrow 0$, this transition would turn into the usual electron cyclotron absorption. Although the absorption in the left polarization is negligible for the most interesting range $\omega \ll \omega_{B e}$, we shall see below that it plays a crucial role for correct evaluation of $\chi_{ \pm 1}^{H}$ and of the NM polarizations in the low-frequency range. The only important transition for the right polarization occurs onto the first excited tightly bound level (010); its oscillator strength, $f_{\kappa \kappa^{\prime}}^{+} \sim 2\left[\gamma^{-1} \ln \gamma+\left(m_{e} / m_{p}\right)\right]$, and transition energy, $\hbar \omega_{\kappa^{\prime} \kappa} \sim$ Ry $\ln \gamma+\hbar \omega_{B p}$, are much smaller than those for $\alpha=-1$. This transition would be the proton cyclotron absorption if Coulomb binding could be neglected. The transitions allowed for the longitudinal polarization proceed onto the hydrogen-like levels of odd parity, creating a series of spectral lines with the leading line formed by the transition to the lowest hydrogen-like level, (000) $\rightarrow(001)$. The oscillator strengths, $f_{\kappa \kappa^{\prime}} \lesssim 1$, and the transition frequencies, $\hbar \omega_{\kappa^{\prime} \kappa} \sim$ Ry $\ln \gamma$, are smaller than for $\alpha=-1$ but substantially greater than for $\alpha=+1$. These transitions, solely caused by the Coulomb binding, disappear when the binding is turned off (if the electron-ion collisions are neglected).

Equations (31)-(33) are obtained in the adiabatic approximation, which leads to some errors in the oscillator strengths. In particular, this approximation implies that $\tilde{\omega}_{\kappa^{\prime} \kappa} \ll \omega_{B e}$ so that one cannot trust second term in the numerator of (31). Indeed, the slightly different approach (still in the frames of the adiabatic approximation) used by Hasegawa \& Howard (1961) leads to the minus sign before this term, instead of plus. Moreover, equations (31) and (32) do not exactly satisfy the sum rule for the circular polarizations (see, e. g., Hasegawa \& Howard 1961),

$$
\sum_{\kappa^{\prime}}\left(f_{\kappa \kappa^{\prime}}^{-1}+f_{\kappa \kappa^{\prime}}^{+1}\right)=2 .
$$

Indeed, the sum of the oscillator strengths (31) and (32) for the two transitions, even without account for, e. g., bound-free transitions for the circular polarizations (see 
below), exceeds 2. Therefore, more accurate values for the oscillator strengths, obtained numerically with allowance for the non-adiabatic corrections, should be used for precise calculations. We shall use below the values from Forster et al. (1984). For the magnetic field $B=2.35 \times 10^{12} \mathrm{G}(\gamma=1000)$ and for the transitions included into our calculations, they are listed in Table 1.

For the bound-free transitions, the final state belongs to the continuum: $\kappa^{\prime}=\left(N^{\prime} s^{\prime} \epsilon^{\prime} \nu^{\prime}\right)$, where $\epsilon^{\prime}(>0)$ is the electron energy in the (one-dimensional) continuum, and $\nu^{\prime}= \pm$ is the parity of the longitudinal function. The sum over the final states in equation (20) is

$$
\sum_{\kappa^{\prime}}=\frac{L_{z} \sqrt{2 m_{e}}}{2 \pi \hbar} \sum_{N^{\prime} s^{\prime} \nu^{\prime}} \int_{0}^{\infty} \frac{d \epsilon^{\prime}}{\sqrt{\epsilon^{\prime}}},
$$

where $L_{z}$ is the the $z$-extension of the periodicity length (the normalization length) of the continuum wave function of the final state. The photoionization cross section is the sum of the partial cross sections

$$
\sigma_{\kappa}^{\alpha}(\omega)=\sum_{N^{\prime} s^{\prime} \nu^{\prime}} \sigma_{\kappa \rightarrow N^{\prime} s^{\prime} \nu^{\prime}}^{\alpha}(\omega)
$$

which differ from zero for frequencies above the photoionization thresholds,

$\omega_{N^{\prime} s^{\prime}}^{(t)} \equiv \epsilon_{000} / \hbar+\left[N^{\prime}\left(1+m_{e} / m_{p}\right)+s^{\prime} m_{e} / m_{p}\right] \omega_{B e}$ if only the ground state is populated. The photoionization cross sections for the atoms at rest have been considered by Potekhin and Pavlov (1993) whose approach and codes we shall use for computations and analysis. In the dipole approximation, only one term contributes to the sum (36) for a given polarization: $N^{\prime}=0, s^{\prime}=0, \nu^{\prime}=-$ for $\alpha=0 ; N^{\prime}=1, s^{\prime}=-1, \nu^{\prime}=+$ for $\alpha=-1$; and $N^{\prime}=0, s^{\prime}=1$, $\nu^{\prime}=+$ for $\alpha=+1$. Then the cross sections can be expressed in a form similar to that for the bound-bound transitions:

$$
\begin{aligned}
\sigma_{\kappa}^{-1}(\omega) & =\pi a_{B}^{2} \alpha_{F} \frac{2 \omega}{\omega_{B e}}\left|\tilde{Z}_{1-1 \epsilon^{\prime}+, 000}^{(0)}\right|^{2} \sqrt{\frac{\mathrm{Ry}}{\hbar\left(\omega-\omega_{t}^{-1}\right)}} \\
\sigma_{\kappa}^{+1}(\omega) & =\pi a_{B}^{2} \alpha_{F} \frac{2 \omega}{\omega_{B e}}\left|\tilde{Z}_{01 \epsilon^{\prime}+, 000}^{(0)}\right|^{2} \sqrt{\frac{\mathrm{Ry}}{\hbar\left(\omega-\omega_{t}^{+1}\right)}} \\
\sigma_{\kappa}^{0}(\omega) & =\pi a_{B}^{2} \alpha_{F} \frac{\hbar \omega}{\mathrm{Ry}}\left|\tilde{Z}_{00 \epsilon^{\prime}-, 000}^{(1)}\right|^{2} \sqrt{\frac{\mathrm{Ry}}{\hbar\left(\omega-\omega_{t}^{0}\right)}},
\end{aligned}
$$

where $\alpha_{F}$ is the fine structure constant,

$$
\tilde{Z}_{N^{\prime} s^{\prime} \epsilon^{\prime} \nu^{\prime}, 000}^{(k)}=\left(L_{z} / a_{B}\right) \int_{-\infty}^{\infty} \mathrm{d} z g_{N^{\prime} s^{\prime} \epsilon^{\prime} \nu^{\prime}}^{*}(z)\left(z / a_{B}\right)^{k} g_{000}(z) \quad(k=0,1),
$$

$\hbar \omega_{t}^{-1}=\epsilon_{000}+\hbar \omega_{B e}, \hbar \omega_{t}^{+1}=\epsilon_{000}+\hbar \omega_{B p}$, and $\hbar \omega_{t}^{0}=\epsilon_{000}$ are the threshold energies, and $\epsilon^{\prime}=\hbar\left(\omega-\omega_{t}^{\alpha}\right)$ in the continuum wave functions $g_{N^{\prime} s^{\prime} \epsilon^{\prime} \nu^{\prime}}(z)$. Similar to the bound-bound transitions, we have $\tilde{Z}_{1-1 \epsilon^{\prime} \nu^{\prime}, 000}^{(0)}=\tilde{Z}_{01 \epsilon^{\prime} \nu^{\prime}, 000}^{(0)}$. It was shown by Hasegawa \& Howard (1961) 
and Schmitt et al. (1981) that the overlapping integrals $\tilde{Z}^{(0)}$ and the matrix elements $\tilde{Z}^{(1)}$ are proportional to $\left(\omega-\omega_{t}^{\alpha}\right)^{1 / 2}$ at $\omega \rightarrow \omega_{t}^{\alpha}+0$, so that the cross sections are finite near the thresholds. The overlapping integrals, and the cross sections for the circular polarizations, are very small because the continuum wave functions are almost orthogonal to the wave function of the ground state. A convenient measure for the strength of the photoionization absorption for a given polarization is the cumulative (integral) bound-free oscillator strength obtained by integration over the final continuum states. From Table 1, where the integral bound-free oscillator strengths are presented together with the photoionization thresholds, we see that the bound-free transitions are much weaker than the bound-bound ones for the circular polarizations, being somewhat stronger than the bound-bound transitions for the longitudinal polarization. Thus, the main transitions are: $(000) \rightarrow\left(00 \epsilon^{\prime}-\right)$ and $(000) \rightarrow(001)$ for $\alpha=0,(000) \rightarrow(1-10)$ for $\alpha=-1$, and $(000) \rightarrow(010)$ for $\alpha=+1$. We include all the transitions listed in Table 1 into our calculations of the absorption coefficients and the components of the polarization tensor. Note that the oscillator strengths in Table 1 satisfy the dipole sum rules with accuracy certainly better than $1 \%$.

\subsection{Broadening Mechanisms and Effects of Finite Atomic Velocities}

The above equations correspond to the case of isolated atoms at rest. They yield infinitely sharp features in both the antihermitian and hermitian parts of the polarizability. Thermal motion of atoms and interaction with surrounding particles broaden and smooth the sharp features. It was shown by Pavlov and Mészáros (1993) that the most important broadening mechanism is associated with different coupling of the internal and center-ofmass motions (the difference between transverse masses, in the perturbation approach) of different levels. In particular, an infinitely narrow bound-bound profile $\delta\left(\omega-\omega_{\kappa^{\prime} \kappa}\right)$ turns, in the perturbation approach, into

$$
\delta\left(\omega-\omega_{\kappa^{\prime} \kappa}\right) \rightarrow \psi_{M}\left(\omega-\omega_{\kappa^{\prime} \kappa}\right) \equiv \begin{cases}\left.\left(\Gamma_{M}\right)^{-1} \exp \left[\omega-\omega_{\kappa^{\prime} \kappa}\right) / \Gamma_{M}\right] & \omega<\omega_{\kappa^{\prime} \kappa} \\ 0 & \omega>\omega_{\kappa^{\prime} \kappa}\end{cases}
$$

The magnetic width $\Gamma_{M}$ is

$$
\Gamma_{M}=\frac{k T}{\hbar}\left(1-\frac{M_{\kappa}^{\perp}}{M_{\kappa^{\prime}}^{\perp}}\right)
$$

where $M_{\kappa}^{\perp}$ is the transverse mass of the level $\kappa$. The magnetic width grows linearly with temperature $\left(\hbar \Gamma_{M} \simeq k T\right.$ for the transitions from the ground level to highly excited states) 
and dominates all other broadening mechanisms. The magnetic broadening also smoothes the photoionization edges:

$$
\mu_{\alpha}^{\mathrm{bf}}(\omega)=n_{a}[1-\exp (-\hbar \omega / k T)] \int_{\max \left(\omega, \omega_{t}^{\alpha}\right)}^{\infty} \mathrm{d} \omega^{\prime} \psi_{M}\left(\omega-\omega^{\prime}\right) \sigma_{\kappa}^{\alpha}\left(\omega^{\prime}\right)
$$

so that the bound-free profiles acquire exponentially decreasing low-frequency tails.

Although the magnetic width is very large, we see from equation (41) that it still retains infinitely sharp high-frequency edges of the bound-bound profiles. In order to avoid logarithmic singularities in $\chi^{H}$, arising after the KK transformation of such functions, one should take into account additional broadening mechanisms: Doppler broadening and/or collisional broadening.

The Doppler width of the spectral lines becomes anisotropic in the presence of strong magnetic field (Pavlov and Mészáros 1993). For a transition $\kappa \rightarrow \kappa^{\prime}$, the Doppler width is

$$
\Gamma_{D}=\alpha_{F} \omega_{\kappa^{\prime} \kappa} \sqrt{\frac{m_{e}}{M} \frac{k T}{\mathrm{Ry}}} \sqrt{\cos ^{2} \theta+\frac{M M_{\kappa}^{\perp}}{\left(M_{\kappa^{\prime}}^{\perp}\right)^{2}} \sin ^{2} \theta},
$$

where $M=m_{p}+m_{e}$ is the usual "longitudinal" mass of the atom, and $\theta$ is the angle between the magnetic field and the wave vector.

Since the density in the neutron star atmospheres is rather high, collisional broadening may be the most significant. The theory of collisional broadening in very strong magnetic fields has not yet been developed. A particular case of the Stark broadening by plasma electrons, which is expected to be the most important in strongly ionized gases, has been considered by Pavlov \& Potekhin (1995) in the impact approximation. In the case of weakly ionized or non-ionized gases the broadening is mainly caused by interactions of neutral particles (e. g., van der Waals interactions). We know from the theory of spectral lines in the non-magnetic case (e. g., Sobelman, Vainshtein \& Yukov 1981) that the most important broadening mechanism for strong transitions in a single-component gas is so-called self-broadening (or resonant broadening). It results in a Lorentz profile with a width that can be estimated as

$$
\Gamma_{s} \sim \frac{e^{2}}{m_{e} \omega_{\kappa^{\prime} \kappa}} f_{\kappa \kappa^{\prime}} n_{a}=4 \frac{\mathrm{Ry}}{\hbar \omega_{\kappa^{\prime} \kappa}} f_{\kappa \kappa^{\prime}}\left(n_{a} a_{B}^{3}\right) \frac{\mathrm{Ry}}{\hbar},
$$

where $f_{\kappa \kappa^{\prime}}$ is the oscillator strength of the transition, and $n_{a}$ is the number density of atoms. We adopt the same estimate for strongly magnetized atoms. The self-broadening width becomes negligibly small for very weak transitions. However, it seems obvious that the collisional width cannot be smaller than $\Gamma_{g}=\Gamma_{g \kappa}+\Gamma_{g \kappa^{\prime}}$, where

$$
\Gamma_{g \kappa} \sim 2 n_{a}\langle\sigma v\rangle \simeq 2 n_{a}\left(\sigma_{\perp \kappa}\left\langle v_{\perp \kappa}\right\rangle+\sigma_{\| \kappa}\left\langle v_{\|}\right\rangle\right)=\frac{4}{\alpha_{F}}\left(n_{a} a_{B}^{3}\right) \frac{\langle\sigma v\rangle}{a_{B}^{2} c} \frac{\mathrm{Ry}}{\hbar}
$$


and $\sigma_{\perp \kappa} \sim 2\left(r_{\kappa}+r_{0}\right)\left(l_{\kappa}+l_{0}\right)$ and $\sigma_{\| \kappa} \sim \pi\left(r_{\kappa}+r_{0}\right)^{2}$ are "geometrical" transverse and longitudinal cross sections for a collision of the atom in the quantum state $\kappa$ with a background atom in the ground state. The cylindrical radius of the atom can be estimated as $r_{\kappa} \sim a_{B}[(|s|+1) / \gamma]^{1 / 2}$; its longitudinal extension is $l_{\kappa} \sim 1.2 a_{B}(|s|+1)^{1 / 4}(\ln \gamma)^{-1}$ for the tightly bound levels, or $l_{\kappa} \sim 1.6 a_{B}\{\operatorname{Int}[(n+1) / 2]\}^{2}$ for the hydrogen-like levels (Potekhin 1994). The transverse and longitudinal mean relative velocities of the colliding atoms are $\left\langle v_{\perp \kappa}\right\rangle=\left(\pi k T / 2 \mu_{\kappa}\right)^{1 / 2}$ and $\left\langle v_{\|}\right\rangle=2(k T / \pi M)^{1 / 2}$, where $\mu_{\kappa}=M_{\perp}^{\kappa} M_{\perp}^{0} /\left(M_{\perp}^{\kappa}+M_{\perp}^{0}\right)$ is the reduced mass. In addition, the lines are broadened by radiative damping (natural broadening). The corresponding widths are negligible for the transitions within the Landau level $N=0$, but the radiative width considerably exceeds the collisional widths for the main transition in the left polarization, $(000) \rightarrow(0-10)$. For this transition we have

$$
\Gamma_{r}=\frac{4 e^{2} \omega_{B e}^{2}}{3 m_{e} c^{3}}=\frac{8}{3} \alpha_{F}^{3} \gamma^{2} \frac{\mathrm{Ry}}{\hbar} .
$$

The collisional and radiative broadening of an infinitely narrow line results in the Lorentz profile

$$
\psi_{L}\left(\omega-\omega_{\kappa^{\prime} \kappa}\right)=\frac{\Gamma_{L}}{2 \pi}\left[\left(\omega-\omega_{\kappa^{\prime} \kappa}\right)^{2}+\frac{\Gamma_{L}^{2}}{4}\right]^{-1} ; \quad \Gamma_{L}=\Gamma_{s}+\Gamma_{g}+\Gamma_{r} .
$$

An example of the values of the magnetic, Lorentz and Doppler widths is presented in Table 1 for the transitions of interest. We see that the Doppler and Lorentz widths are generally comparable. Nevertheless, we shall neglect Doppler broadening in our calculations. The convolution of the Doppler and Lorentz profiles coincides with the Lorentz profile far from the line center (at $\left|\omega-\omega_{\kappa^{\prime} \kappa}\right| \gtrsim \Gamma_{D}$ ), and the resolution in the calculations presented here is not sufficient to describe the details near the line cores. Moreover, the above estimates of the widths are very approximate, and the primary object of introducing the broadening is to avoid sharp edges in the absorption coefficients. Thus, we assume that the bound-bound lines are broadened by magnetic and Lorentz broadening:

$$
\mu_{\alpha}^{\mathrm{bb}}(\omega)=\frac{2 \pi^{2} e^{2} n_{a}}{m_{e} c}\left[1-\exp \left(-\frac{\hbar \omega}{k T}\right)\right] \sum_{\kappa^{\prime}} f_{\kappa \kappa^{\prime}}^{\alpha} \int_{\omega}^{\infty} \mathrm{d} \omega^{\prime} \psi_{M}\left(\omega-\omega^{\prime}\right) \psi_{L}\left(\omega^{\prime}-\omega_{\kappa^{\prime} \kappa}\right) .
$$

\section{RESULTS}

\subsection{Components of the Polarizability Tensor}

As a representative example, we investigated the NM properties for conditions typical for photospheres of moderately old, isolated neutron stars (Pavlov et al. 1995ab): 
$B=2.35 \times 10^{12} \mathrm{G}\left(\gamma=1000, \hbar \omega_{B e}=2000 \mathrm{Ry}, \hbar \omega_{B p}=1.09 \mathrm{Ry}\right), k T=1 \mathrm{Ry}$ $\left(T=1.58 \times 10^{5} \mathrm{~K}\right), \rho=1 \mathrm{~g} \mathrm{~cm}^{-3}\left(n_{a} a_{B}^{3}=0.0885\right)$. The ionization energy of hydrogen in this field is $15.32 \mathrm{Ry}$. All the relevant bound-bound and bound-free transitions, the oscillator strengths, and the widths are listed in Table 1 . Although the problem of ionization equilibrium in strongly magnetized, dense hydrogen has not been satisfactorily solved so far, preliminary estimates show that the ionization degree is expected to be low at this relatively low temperature and high density, which allows us to neglect the ionized component and to demonstrate qualitative features of the NMs in a nonionized, strongly magnetized gas.

Three absorption coefficients $\mu_{\alpha}(\omega)$, calculated as described in Sections 2 and 3, are shown in Figure 1, and the corresponding antihermitian components of polarizability tensor $\chi_{\alpha}^{A}(\omega)=(c / 4 \pi \omega) \mu_{\alpha}(\omega)$ are shown in Figure 2. We see that the behavior of $\mu_{\alpha}$ and $\chi_{\alpha}^{A}(\omega)$ is quite different for different $\alpha$. For $\alpha=-1$, it has one very strong resonance associated with the bound-bound transition $(000) \rightarrow(1-10)$ and centered very close to the electron cyclotron frequency. The strongest absorption in the most interesting frequency range $\omega \ll \omega_{B e}$ is that for radiation polarized along the field $(\alpha=0)$. It appears as a broad resonance around $\approx 15 \mathrm{Ry}$ with a fine structure near the maximum associated with the peaks of the overlapped bound-bound and bound-free absorption. The high-energy tail of the resonance is due the photoionization $(000) \rightarrow\left(00 \epsilon^{\prime}-\right)$, and the low-energy tail is caused by the magnetic broadening of the bound-bound transitions [mainly, of $(000) \rightarrow(001)$ ]. Two features are seen in $\mu_{+1}(\omega)$ and $\chi_{+1}^{A}(\omega)$ : the broadened, asymmetric spectral line $(000) \rightarrow(010)$ and the (much weaker) photoionization spectrum $(000) \rightarrow\left(01 \epsilon^{\prime}+\right)$.

If the gas were fully ionized, the $\chi_{-1}^{A}$ component would be almost the same - the peak would shift from 2004 Ry to the electron cyclotron resonance at 2000 Ry. The $\chi_{+1}^{A}$ component would turn into a narrow peak at the proton cyclotron frequency, the width of the resonance being determined by the proton-proton collisions (free charged particles are not subject to magnetic broadening - their "transverse masses" are infinite). The longitudinal component $\chi_{0}^{A}$ would decrease monotonically with $\omega$ (the only "resonance" lies at $\omega=0$ for the unbound particles), describing the inverse bremsstrahlung due to the electron-ion collisions. Thus, the Coulomb binding essentially alters the antihermitian part of the polarization tensor, especially for the longitudinal component.

We made the KK transformation of $\omega \chi_{\alpha}^{A}$ (see eq. [19]) and obtained the hermitian components $\chi_{\alpha}^{H}(\omega)$ which are shown in Figure 2 for $\omega>0$. The accuracy of the numerical KK transformation was tested with a simple model of fully ionized plasma. As expected, the hermitian part has spectral features associated with those of the antihermitian part. Indeed, as follows from equations (18) and (19), an absorption feature of $\chi_{\alpha}^{A}(\omega)$ centered at a positive frequency $\omega_{\alpha}$, generates the contribution to $\chi_{\alpha}^{H}(\omega)$ equal to 
$\omega_{p a}^{2} f^{\alpha}\left[8 \pi \omega_{\alpha}\left(\omega_{\alpha}-\omega\right)\right]^{-1}$ if the distance $\left|\omega-\omega_{\alpha}\right|$ from the feature is much larger than its effective width, and $\exp \left(-\hbar \omega_{\alpha} / k T\right) \ll 1$. Each spectral feature at the positive frequency $\omega_{\alpha}$ has its "counterpart" at the negative frequency $-\omega_{-\alpha}$; e. g., for the spectral line $(000) \rightarrow(010)$ in the right polarization $\left(\omega_{+1}=\omega_{010,000}, f^{+1}=f_{000,010}^{+1}\right)$ the counterpart line is centered at $-\omega_{-1}=-\omega_{1-10,000}$, and its oscillator strength is $f^{-1}=f_{000,1-10}^{-1}$. This counterpart generates $\omega_{p a}^{2} f^{-\alpha}\left[8 \pi \omega_{-\alpha}\left(\omega_{-\alpha}+\omega\right)\right]^{-1}$. Summing these two terms with allowance for the property $\omega_{\alpha} f^{-\alpha}=\omega_{-\alpha} f^{\alpha}$ (see eqs. [17] and [21]), we obtain the total contribution of the feature to the hermitian part:

$$
\chi_{\alpha}^{H(\mathrm{bb})}(\omega)=\frac{\omega_{p a}^{2}}{8 \pi} \frac{f^{\alpha}+f^{-\alpha}}{\left(\omega_{\alpha}-\omega\right)\left(\omega_{-\alpha}+\omega\right)} .
$$

This expression generalizes the well-known result (e. g., Davydov 1976) of the isotropic case, when $\omega_{-1}=\omega_{+1}=\omega_{0}$ and $f^{-1}=f^{+1}=f^{0}$ are the frequency and the oscillator strength of a given transition. Notice that equation (50) describes also the polarizability of a "cold", magnetoactive plasma (e. g., Ginzburg 1970) if $\omega_{p a}=\omega_{p e}=\left(4 \pi n_{e} e^{2} / m_{e}\right)^{1 / 2}$ is the electron plasma frequency, $\omega_{-1}=\omega_{B e}, \omega_{+1}=\omega_{B p}, \omega_{0}=0, f^{-1}=2, f^{+1}=2 m_{e} / m_{p}$, and $f^{0}=1$. In our case of the cold, nonionized gas, $\chi_{ \pm 1}$ is determined by the bound-bound transitions, so that equation (50) immediately gives the circular components of the hermitian part:

$$
\chi_{ \pm 1}^{H}(\omega) \simeq \frac{\omega_{p a}^{2}}{4 \pi\left(\omega_{-} \pm \omega\right)\left(\omega_{+} \mp \omega\right)},
$$

where $\omega_{+} \equiv \omega_{010,000}$ and $\omega_{-} \equiv \omega_{1-10,000}$. The only difference from the corresponding components for the fully ionized plasma is that we replace $\omega_{B p}$ by $\omega_{+}$, and $\omega_{B e}$ by $\omega_{-}$.

Interaction of the longitudinally polarized radiation with the cold gas is mainly determined by the spectral line $(000) \rightarrow(001)$ and the photoionization $(000) \rightarrow(00 \epsilon-)$. The former yields

$$
\chi_{0}^{H(\mathrm{bb})}=\frac{\omega_{p a}^{2} f_{0}}{4 \pi\left(\omega_{0}^{2}-\omega^{2}\right)},
$$

where $f_{0} \equiv f_{000,001}^{0}$ and $\omega_{0} \equiv \omega_{001,000}$. Accurate values for the bound-free contribution can be obtained only numerically. A qualitatively correct behavior of $\chi_{0}^{(b f)}$ can be obtained from an approximate model cross section:

$$
\sigma^{0}(\omega) \approx \sigma_{t}\left(\omega_{t} / \omega\right)^{3} \Theta\left(\omega-\omega_{t}\right)
$$

where $\omega_{t}$ is the threshold frequency and $\sigma_{t}$ is the threshold cross section. Substituting this model expression into equations (19) and (20), we obtain

$$
\chi_{0}^{H(\mathrm{bf})} \simeq \frac{\omega_{p a}^{2} f_{(\mathrm{bf})}^{0}}{4 \pi \omega^{2}}\left[\left(\frac{\omega_{t}}{\omega}\right)^{2} \ln \frac{\omega_{t}^{2}}{\left|\omega_{t}^{2}-\omega^{2}\right|}-1\right],
$$


where $f_{(\mathrm{bf})}^{0}$ is the integral oscillator strength of the bound-free absorption. At $\omega \ll \omega_{t}$, the polarizability resembles that for the bound-bound transition with $\omega_{0} \rightarrow(3 / 2)^{1 / 2} \omega_{t}$, $f^{0} \rightarrow(3 / 4) f_{(\mathrm{bf})}^{0}:$

$$
\chi_{0}^{H(\mathrm{bf})} \simeq \frac{3 \omega_{p a}^{2} f_{(\mathrm{bf})}^{0}}{16 \pi\left(\frac{3}{2} \omega_{t}^{2}-\omega^{2}\right)} .
$$

When $\omega$ approaches $\omega_{t}$, the polarizability diverges logarithmically:

$$
\chi_{0}^{H(\mathrm{bf})} \simeq \frac{\omega_{p a}^{2} f_{(\mathrm{bf})}^{0}}{4 \pi \omega_{t}^{2}} \ln \frac{\omega_{t}}{2\left|\omega_{t}-\omega\right|} .
$$

At $\omega>\omega_{t}$, it first decreases, changes its sign at $\omega \simeq 1.16 \omega_{t}$, reaches a minimum at $\omega \simeq 1.58 \omega_{t}$, and approaches zero at $\omega \gg \omega_{t}$ :

$$
\chi_{0}^{H(\mathrm{bf})} \simeq \frac{\omega_{p a}^{2} f_{(\mathrm{bf})}^{0}}{4 \pi\left[\omega_{t}^{2} \ln \left(\omega^{2} / \omega_{t}^{2}\right)-\omega^{2}\right]} .
$$

Equations (54) through (57) are not applicable too close to $\omega_{+}(=5.14 \mathrm{Ry}), \omega_{0}$ (=14.4 Ry), $\omega_{t}$ (=15.3 Ry), and $\omega_{-}(=2004 \mathrm{Ry})$, where the bound-bound or bound-free polarizabilities diverge if broadening mechanisms are not included. The magnetically broadened spectral line generates the following longitudinal component of the hermitian part of the polarizability $\left(\right.$ at $\left.\exp \left(-\hbar \omega_{0} / k T\right) \ll 1\right)$

$$
\chi_{0}^{H(\mathrm{bb})}=\frac{\omega_{p a}^{2} f_{0}}{8 \pi \omega \Gamma_{M}}\left[\exp \left(-\frac{\omega_{0}-\omega}{\Gamma_{M}}\right) \operatorname{Ei}\left(\frac{\omega_{0}-\omega}{\Gamma_{M}}\right)-\exp \left(-\frac{\omega_{0}+\omega}{\Gamma_{M}}\right) \operatorname{Ei}\left(\frac{\omega_{0}+\omega}{\Gamma_{M}}\right)\right],
$$

where $\operatorname{Ei}(x)$ is the exponential-integral function. At $\left|\omega_{0}-\omega\right| \gg \Gamma_{M}$ this equation turns into equation (52) with a shifted central frequency:

$$
\chi_{0}^{H(\mathrm{bb})}=\frac{\omega_{p a}^{2} f_{0}}{4 \pi\left[\left(\omega_{0}-\Gamma_{M}\right)^{2}-\omega^{2}\right]} .
$$

Near the central frequency of the unbroadened line, the polarizability diverges logarithmically:

$$
\chi_{0}^{H(\mathrm{bb})}=-\frac{\omega_{p a}^{2} f_{0}}{8 \pi \omega_{0} \Gamma_{M}}\left[\ln \frac{\Gamma_{M}}{\left|\omega_{0}-\omega\right|}-C\right],
$$

where $C \simeq 0.577$ is the Euler constant. Thus, with frequency growing from zero, the polarizability generated by a magnetically broadened, isolated spectral line first grows, reaches its maximum at $\omega \simeq \omega_{0}-1.35 \Gamma_{M}$, changes its sign at $\omega \simeq \omega_{0}-0.37 \Gamma_{M}$, reaches its logarithmically infinite minimum at $\omega=\omega_{0}$, and then grows steadily to zero. 
The magnetically broadened circular components are given by similar equations,

$$
\chi_{ \pm 1}^{H(\mathrm{bb})}=\frac{\omega_{p a}^{2}}{8 \pi \omega}\left[\frac{f_{ \pm}}{\Gamma_{M \pm}} \exp \left(\frac{\omega-\omega_{ \pm}}{\Gamma_{M \pm}}\right) \operatorname{Ei}\left(\frac{\omega_{ \pm}-\omega}{\Gamma_{M \pm}}\right)-\frac{f_{\mp}}{\Gamma_{M \mp}} \exp \left(-\frac{\omega_{\mp}+\omega}{\Gamma_{M \mp}}\right) \operatorname{Ei}\left(\frac{\omega_{\mp}+\omega}{\Gamma_{M \mp}}\right)\right],
$$

which turn into equations (51) far from the frequencies $\omega_{ \pm}$. In the most interesting region $\omega \ll \omega_{B e}$, the left-circular component varies very smoothly,

$$
\chi_{-1}^{H(\mathrm{bb})}=\frac{\omega_{p a}^{2}}{4 \pi \omega_{-}\left(\omega_{+}+\omega\right)}
$$

whereas the right-circular component shows a resonant behavior near $\omega_{+}$, similar to the above-described behavior of the longitudinal component around $\omega_{0}$.

Magnetic broadening of the bound-free polarizability does not change the polarizability at $\left|\omega-\omega_{t}\right| \gg \Gamma_{M}$ (see eqs. [41], [55], and [57]), and it removes the logarithmic divergence at $\omega_{t}$ :

$$
\chi_{0}^{H(\mathrm{bf})} \sim \frac{\omega_{p a}^{2} f_{\mathrm{bf}}^{0}}{4 \pi \omega_{t}^{2}} \ln \frac{\omega_{t}}{\Gamma_{M}} \quad \text { at } \quad \omega \simeq \omega_{t} .
$$

The logarithmic divergence of the magnetically broadened bound-bound polarizability (see eq. [60) is removed by the additional Lorentz (or Doppler) broadening. The polarizability near the line center can be estimated as

$$
\chi_{0}^{H(\mathrm{bb})} \sim-\frac{\omega_{p a}^{2} f_{0}}{8 \pi \omega_{0} \Gamma_{M}} \ln \frac{\Gamma_{M}}{\Gamma_{L}}
$$

for the longitudinal component. A similar estimate is true for the right component, with replacing $\omega_{0} \rightarrow \omega_{+}$and $f_{0} \rightarrow f_{+}$.

The above equations completely describe qualitative properties of the cyclic components of the polarizability tensor. The results of numerical calculation are shown in Figure 2.

\subsection{Polarization Properties of the Normal Modes}

The NM polarizations can be conveniently described by the parameters $q$ and $p$ defined in equations (8) and (12). In wide frequency ranges far from the bound-bound resonances and the bound-free thresholds, the hermitian part of the polarizability substantially exceeds the antihermitian part, so that $|q| \gg|p|$, and the polarizations are determined by the parameter

$$
q=\tilde{q} \frac{\sin ^{2} \theta}{2 \cos \theta}, \quad \text { where } \quad \tilde{q} \simeq \frac{2 \chi_{0}^{H}-\chi_{+}^{H}-\chi_{-}^{H}}{\chi_{+}^{H}-\chi_{-}^{H}} .
$$


In the case of fully ionized plasma, we have

$$
\tilde{q}=\frac{\omega^{2}\left(\omega_{B p}^{2}+\omega_{B e}^{2}-\omega_{B p} \omega_{B e}\right)-\omega_{B p}^{2} \omega_{B e}^{2}}{\omega^{3}\left(\omega_{B e}^{2}-\omega_{B p}^{2}\right)} .
$$

At $\omega \gg \omega_{B p}$ only the electron component is important, and $\tilde{q}=\omega_{B e} / \omega$ is a smooth function of frequency. At $\omega \gg \omega_{B e}$ the parameter $\tilde{q} \ll 1$, that is, the NMs are circularly polarized in a wide range of angles $\theta$. With decreasing frequency, $\tilde{q}$ increases (the polarization ellipses become narrower) and reaches its maximum, $\tilde{q}_{\max } \simeq 2 \omega_{B e} /\left(3^{3 / 2} \omega_{B p}\right) \simeq 707$ at $\omega=\sqrt{3} \omega_{B p}$, where the NM polarization is virtually linear; then it sharply decreases, crosses zero in the very vicinity of proton cyclotron resonance, at $\omega \simeq \omega_{B p}\left(1+2 \omega_{B p} / \omega_{B e}\right) \simeq 1.0011 \omega_{B p}$ and tends to $-\infty\left(\tilde{q} \simeq-\omega_{B e} \omega_{B p}^{2} / \omega^{3}\right.$ at $\left.\omega \rightarrow 0\right)$. The fact that $\tilde{q}$ is very small near the proton cyclotron resonance means that one should expect a peculiar behavior of the NMs there; in particular, strong non-orthogonality of the NMs at some angles. To analyze the NM properties around the $q=0$ point, one needs to calculate the parameter $p$ whose behavior depends on the absorption mechanism operating at these frequencies.

The relatively simple behavior of $q$ and $p$ for the fully ionized plasma is due to the absence of resonances of the longitudinal component of the polarizability $\chi_{0}$ at finite frequencies. For the non-ionized gas, these resonances do exist due to Coulomb binding, which strongly complicates the picture (see Fig. 3). Nevertheless, even in this case the NM properties can be investigated qualitatively with the aid of approximate expressions for $\chi_{\alpha}$ derived above. At frequencies well above the photoionization threshold $\omega_{t}(z 20-30$ Ry in our example), we obtain from equations (51), (57), and (52),

$$
\tilde{q}=\frac{\omega_{-}}{\omega}\left[1+\frac{f_{0} \omega_{0}^{2}+f_{\mathrm{bf}} \omega_{t}^{2} \ln \left(\omega^{2} / \omega_{t}^{2}\right)}{\omega^{2}}\right] .
$$

We see that the correction for the longitudinal absorption/refraction increases the value of $\tilde{q}$ as compared to the fully ionized case, i. e., the NM polarization is more linear than in the fully ionized plasma. At frequencies much lower than $\omega_{0}-\Gamma_{M}(\lesssim 12$ Ry in our example), and not too close to $\omega_{+}$, we obtain from equations (51), (55), and (52),

$$
\tilde{q}=\frac{\omega_{-}\left(\omega_{+}^{2}-\omega^{2}\right)}{\omega}\left[\frac{f_{0}}{\left(\omega_{0}-\Gamma_{M}\right)^{2}-\omega^{2}}+\frac{3 f_{\mathrm{bf}}}{2\left(3 \omega_{t}^{2}-2 \omega^{2}\right)}\right] .
$$

At frequencies much below $\omega_{+}$, we have $\tilde{q} \propto \omega_{-} / \omega$, as at high frequencies (eq. 67), but this ratio is now multiplied by a small factor $\sim f_{0} \omega_{+}^{2} / \omega_{0}^{2}$. Due the Coulomb binding, the behavior of $\tilde{q}$ at these low frequencies is qualitatively different from that in the fully ionized plasma. The function $\tilde{q}(\omega)$ changes its sign near $\omega_{+}$and keeps falling with increasing $\omega$ until $\omega$ approaches $\omega_{0}-\Gamma_{M}$. Equation (68) is not accurate near $\omega_{+}$, where absorption plays 
a role and the antihermitian component $\chi_{+1}^{A}$ should be taken into account. Nevertheless, it satisfactorily describes the qualitative behavior of $q$ even in this region.

It is clear from equations (67) and (68) that $q(\omega)$ must change its sign at least once more, in a region between $\sim\left(\omega_{0}-\Gamma_{M}\right)$ and $\sim \omega_{t}$. The behavior of $q(\omega)$ in this region is determined by interplay of the bound-bound and bound-free refraction and absorption (both hermitian and antihermitian components are important), the details depending on the broadening mechanisms and the value of the magnetic field. Since $\tilde{q} \simeq 2 \chi_{0}^{H} /\left(\chi_{+1}^{H}-\chi_{-1}^{H}\right)$ in this region, and $\chi_{+1}^{H}-\chi_{-1}^{H}(<0)$ is a smooth function of frequency, the shape of $q(\omega)$ reproduces that of $-\chi_{0}$ (cf. Fig. 2). In particular, near the center of an isolated line, $\tilde{q}=\tilde{q}_{\min } \simeq-0.4 f_{0} \omega_{-} / \Gamma_{M}$ at $\omega \simeq \omega_{0}-1.4 \Gamma_{M}$, and $\tilde{q}=\tilde{q}_{\max } \sim\left(\omega_{-} / 2 \Gamma_{M}\right) \ln \left(\Gamma_{M} / \Gamma_{L}\right)$ at $\omega \simeq \omega_{0}$. In our example, $\tilde{q}$ reaches its minimum, $\simeq-237$, at $\hbar \omega=13.35 \mathrm{Ry}$, then sharply grows, changes its sign at $\hbar \omega=14.22 \mathrm{Ry}$, reaches the maximum, $\simeq 472$, at $\hbar \omega=14.42 \mathrm{Ry}$, and then, after some ups and downs associated with the longitudinal bound-free transition, approaches the asymptotic curve (67).

The parameter $p$, which is associated with absorption of radiation (antihermitian components), is important whenever $|p|$ exceeds $|q|$, that is, in a very narrow range near $\omega_{+}$, and in the range of strong longitudinal absorption $(\sim 13-20$ Ry in our example). Around $\omega_{+}$, the antihermitian part is determined by the right-polarization absorption, $\chi_{+1}^{A} \gg \chi_{0}^{A}, \chi_{-1}^{A}$, and $\chi_{+1}^{H} \gg \chi_{-1}^{H}$. In this range

$$
\tilde{p} \equiv p \frac{2 \cos \theta}{\sin ^{2} \theta} \simeq-\frac{2 \chi_{+1}^{A}\left(\chi_{0}^{H}-\chi_{+1}^{H}\right)}{\left(\chi_{+1}^{H}\right)^{2}+\left(\chi_{+1}^{A}\right)^{2}} .
$$

The ratio $\left.\chi_{+1}^{A} /\left[\left(\chi_{+1}^{H}\right)^{2}+\chi_{+1}^{A}\right)^{2}\right]$ is a smooth function of frequency (the resonances in the numerator and denominator cancel each other), and so is $\chi_{0}^{H}$. In our example, $\chi_{0}^{H}$ exceeds $\left|\chi_{+1}^{H}\right|$ in this frequency range (see Fig. 2), and $\tilde{p}$ decreases with decreasing $\omega$ almost smoothly, with a weak wiggle around 5.1 Ry caused by nonmonotonic behavior of the relatively small $\chi_{+1}^{H}$. A characteristic value of $\tilde{p}$ in this range can be roughly estimated as $\tilde{p} \sim f_{0} \omega_{-} \Gamma_{M+} /\left(\omega_{0}^{2}-\omega_{+}^{2}\right)(\sim 1$ in our example - see Fig. 3. $)$.

In the range of strong longitudinal absorption (and, more generally, at $\omega_{+} \ll \omega \ll \omega_{-}$), we can neglect $\chi_{ \pm 1}^{A}$, so that

$$
\tilde{p} \simeq \frac{2 \chi_{0}^{A}}{\chi_{+1}^{H}-\chi_{-1}^{H}} \simeq-\frac{2 c \omega_{-} \mu_{0}(\omega)}{\omega_{p a}^{2}},
$$

and the dependence $p(\omega)$ reproduces that of the longitudinal absorption coefficient $\mu_{0}(\omega)$. In particular, characteristic values of $\tilde{p}$ near $\omega_{0}$ are $\sim f_{0} \omega_{-} / \Gamma_{M} \sim \tilde{q}(\sim 500$ in our example). They are considerably greater than those near $\omega_{+}$, which results in different behavior of the NM polarizations near these frequencies. 
In a wide range of frequencies, where $|q| \gg|p|(\lesssim 3 \mathrm{Ry}$, between $\simeq 6$ and $\simeq 10 \mathrm{Ry}$, and at $\gtrsim 30 \mathrm{Ry}$, in our example), the NMs are nearly orthogonal:

$$
\delta_{j}=0, \pm \frac{\pi}{2} ; \quad \mathcal{P}_{j}= \pm \frac{1}{|q|+\left(q^{2}+1\right)^{1 / 2}}= \pm \frac{2 \cos \theta}{|\tilde{q}| \sin ^{2} \theta+\left(\tilde{q}^{2} \sin ^{4} \theta+4 \cos ^{2} \theta\right)^{1 / 2}} .
$$

In the most important domain, $\omega \ll \omega_{-}$, we have $|\tilde{q}| \gg 1$ in the wide frequency range, i. e., the NMs are linearly polarized, $\left|\mathcal{P}_{j}\right| \ll 1$, at $\sin \theta \gg(2 / \tilde{q})^{1 / 2}$. In the ranges of strong longitudinal or right-polarization absorption, $q$ and $p$ become comparable, and behavior of the NM polarizations is more complicated. The most peculiar behavior occurs near the critical frequencies where $q=0\left(\hbar \omega_{c 1}=4.751 \mathrm{Ry}\right.$ and $\hbar \omega_{c 2}=14.22 \mathrm{Ry}$ in our example). For each of these frequencies, there exists an angle $\theta_{c}$ such that $\left|\tilde{p}\left(\omega_{c}\right)\right| \sin ^{2} \theta_{c} /\left(2 \cos \theta_{c}\right)=1$; the NM polarizations at $\omega=\omega_{c}$ and $\theta=\theta_{c}$ are completely linear, $\mathcal{P}_{j}=0$, and coincide with each other, $\delta_{1}=\delta_{2}= \pm \pi / 4$ (complete non-orthogonality of NMs). In our example, $\tilde{p}\left(\omega_{c 1}\right)=-3.63$ and $\tilde{p}\left(\omega_{c 2}\right)=-562$, so that the non-orthogonality angles are $\theta_{c 1} \simeq 40.35^{\circ}$ and $\theta_{c 2}=3.41^{\circ}$. Since $\left|p\left(\omega_{c}, \theta\right)\right|<1$ at $\theta<\theta_{c}$, the circular component of polarization is present, $\mathcal{P}_{j}= \pm[(1-|p|) /(1+|p|)]^{1 / 2}$, but the orientations of the polarization ellipses coincide, $\delta_{1}=\delta_{2}= \pm \pi / 4$. If $\theta>\theta_{c}$ (i. e., $\left|p\left(\omega_{c}, \theta\right)\right|>1$ ), then the NMs are linearly polarized, $\mathcal{P}_{j}=0$, but the polarization directions neither coincide nor are orthogonal, $\tan 2 \delta_{j}= \pm\left(p^{2}-1\right)^{-1 / 2}$. The non-orthogonality points and the curves $|q|=1$ and $|p|=1$ in the $\omega-\theta$ plane are shown in Figure 4.

In Figure 5 we demonstrate the frequency dependence of the ellipticities and position angles for three angles of propagation, $\theta=1^{\circ}, 30^{\circ}$, and $75^{\circ}$, so that we cover three regions: $\theta<\theta_{c 2}, \theta_{c 2}<\theta<\theta_{c 1}$, and $\theta>\theta_{c 1}$. In the case of propagation nearly along the magnetic field, the NMs are polarized circularly. The ellipticity $\mathcal{P}_{j}$ decreases slightly in the range around 15 Ry where $p$ is comparable to $q$. The position angles $\delta_{j}$ coincide at the two critical frequencies $\omega_{c 1}$ and $\omega_{c 2}$. Just above the second critical frequency the position angle varies strongly with frequency due to the presence of bound-bound structure in $p$ (see Fig. 3). For the intermediate angle $\theta=30^{\circ}$, the polarization of the NMs becomes linear $\left(\mathcal{P}_{j}=0\right)$ at the second critical frequency, while at the first one it is elliptical with coinciding positional angles. The maximum ellipticity around $5 \mathrm{Ry}$ is due to the fact that in this region $p$ is comparable to $q$, which increases the circular component of polarization. The polarizations of the NMs for $\theta=75^{\circ}>\theta_{c 1}$ are close to linear. They become exactly linear at the two critical frequencies $\omega_{c 1}$ and $\omega_{c 2}$. Around $\omega_{c 1}$, however, the polarization of the NMs is elliptical, since there is still a significant range where $|q|$ is small and $|p|$ is close to unity. This is also the cause of the small non-orthogonality of the NMs around $\omega_{c 1}$. Around $\omega_{c 2}$, the NMs are polarized linearly and are orthogonal because $|p|$ is large and the curve $q(\omega)$ passes the range $|q| \ll 1$ very rapidly.

The general picture is strikingly different from the case of fully ionized plasma, in 
which only one critical frequency exists in the vicinity of $\omega_{B p}$. The Coulomb binding not only shifts this point to higher frequencies and changes the corresponding value of $\theta_{c}$, but also creates at least one more critical frequency in the range of strong longitudinal absorption. Furthermore, it diminishes $\left|\mathcal{P}_{j}\right|$ at high frequencies, $\gtrsim 30$ Ry, and increases it at low frequencies, $\lesssim 5$ Ry.

The location of the critical points where the polarization modes acquire the abovedescribed peculiarities is a sensitive function of the temperature and density because it depends strongly on the shape of the absorption features.

\subsection{Absorption Coefficients of the Normal Modes}

It follows from the above analysis that in the vicinities of the two points $\left(\omega_{c 1}, \theta_{c 1}\right)$ and $\left(\omega_{c 2}, \theta_{c 2}\right)$ in the $\omega-\theta$ plane, the NMs are strongly non-orthogonal (see Fig. 4). Far from these non-orthogonality domains, the NM absorption coefficients $\mu_{j}(j=1,2)$ can be expressed as linear combinations of the 'basic' coefficients $\mu_{\alpha}$ (e. g., Kaminker et al. 1982):

$$
\mu_{j}=\sum_{\alpha=-1}^{+1} a_{j}^{\alpha} \mu_{\alpha}
$$

where

$$
\begin{gathered}
a_{j}^{0}=\frac{\sin ^{2} \theta}{2}\left[1+(-1)^{j} \frac{q}{\sqrt{1+q^{2}}}\right], \\
a_{j}^{ \pm 1}=\frac{1}{4}\left[1+\cos ^{2} \theta \pm(-1)^{j} \frac{2 \cos \theta-q \sin ^{2} \theta}{\sqrt{1+q^{2}}}\right] .
\end{gathered}
$$

As we see from Figure $4,|q| \gg 1$ (the NM polarizations are nearly linear) in a wide domain of angles $\theta$ and frequencies $\omega \ll \omega_{B e}$. For this domain we obtain:

$$
\mu_{1}=\frac{\mu_{+1}+\mu_{-1}}{2}+\frac{\mu_{0} \cos ^{2} \theta}{\tilde{q}^{2} \sin ^{2} \theta} ; \quad \mu_{2}=\mu_{0} \sin ^{2} \theta+\frac{\mu_{+1}+\mu_{-1}}{2} \cos ^{2} \theta
$$

The 'ordinary mode' $j=2$ is polarized in the B-q plane. For purely transverse propagation, $\theta=90^{\circ}$, it is polarized along the magnetic field, so that $\mu_{2}=\mu_{0}$. Since $\mu_{-1} \ll \mu_{+1}$ at

$\omega \ll \omega_{B e}$ (see Fig. 1), the admixture of the transverse-polarization absorption at $\cos \theta \neq 0$ is determined by the right-circular absorption coefficient $\mu_{+1}$. The admixture term is negligibly small in the domain where equations (75) are applicable, except for a narrow frequency range around the resonance of $\mu_{+1}(\sim 2-5$ Ry in our case). The 'extraordinary mode' $j=1$ is polarized perpendicular to the magnetic field, and $\mu_{1}=\mu_{+1} / 2$ at $\theta=90^{\circ}$. 
Since $\mu_{0} \gg \mu_{+1}$ in a wide frequency range, the second term in the equation for $\mu_{1}$, which describes the admixture of the longitudinal-polarization absorption, may substantially exceed the first term at $\cos \theta \neq 0$, in spite of the fact that it is proportional to a small factor $\tilde{q}^{-2}$.

At $\sin \theta \ll \tilde{q}^{-1 / 2}$ in a wide frequency range, and at $\sin \theta \ll \sin \theta_{c}$ near the critical frequencies, the NM polarizations are nearly circular $(|q| \ll 1$ and $|p| \ll 1)$. In this domain,

$$
\begin{aligned}
& \mu_{1}=\mu_{-1}\left[\frac{(1+\cos \theta)^{2}}{4}-\frac{\tilde{q} \sin ^{4} \theta}{8 \cos \theta}\right]+\mu_{0} \frac{\sin ^{2} \theta}{2}+\mu_{+1}\left[\frac{(1-\cos \theta)^{2}}{4}+\frac{\tilde{q} \sin ^{4} \theta}{8 \cos \theta}\right], \\
& \mu_{2}=\mu_{+1}\left[\frac{(1+\cos \theta)^{2}}{4}-\frac{\tilde{q} \sin ^{4} \theta}{8 \cos \theta}\right]+\mu_{0} \frac{\sin ^{2} \theta}{2}+\mu_{-1}\left[\frac{(1-\cos \theta)^{2}}{4}+\frac{\tilde{q} \sin ^{4} \theta}{8 \cos \theta}\right] .
\end{aligned}
$$

For purely longitudinal propagation, $\theta=0$, the NM polarizations are exactly circular, so that $\mu_{1}=\mu_{-1}$ and $\mu_{2}=\mu_{+1}$. The left-circular absorption is very weak; in fact, at $\omega \ll \omega_{B e}$, it is determined by violation of the dipole selection rules due to the motion of atoms in the magnetic field (Pavlov \& Potekhin 1995), which is neglected in our perturbation approach. However, even at very small $\theta \neq 0$, absorption in the $j=1$ mode is mainly determined by the admixture of the (relatively strong) right-circular and longitudinal absorptions, $\mu_{1} \simeq\left(\mu_{+1} / 8\right) \sin ^{4} \theta+\left(\mu_{0} / 2\right) \sin ^{2} \theta$, and the exact value of $\mu_{-1}$ is not important.

Equations (75), (76), and (77) allow one to understand qualitatively behavior of the spectral and angular dependences of $\mu_{j}$ depicted in Figures 6, 7, and 8. Figure 6 demonstrates evolution of $\mu_{j}(\omega)$ with increasing $\theta$ for directions of propagation close to the magnetic field. For $\theta \ll \theta_{c 2}\left(\theta=1^{\circ}\right.$ in Fig. 6), the NMs are polarized almost exactly circularly at all the frequencies. The absorption coefficient of the $j=2$ mode (dashed curve) is determined by the right-circular absorption $\mu_{+1}$ (dominates at $\hbar \omega \lesssim 8 \mathrm{Ry}$ [bound-bound transition to (010)] and $\gtrsim 15 \mathrm{Ry}$ [bound-free transition to $(01 \epsilon+)]$ - cf. Fig. 1) and by a small addition, $\left(\mu_{0} / 2\right) \sin ^{2} \theta$ of the longitudinal absorption (dominates at the longitudinal resonance, $\hbar \omega \sim 10-15 \mathrm{Ry}$ ). The other mode is solely determined (at this angle) by the same contribution of the longitudinal absorption. The absorption coefficients $\mu_{1}$ and $\mu_{2}$ almost coincide with each other at the region of strong longitudinal absorption.

The increase of $\theta$ towards and beyond the critical value $\theta_{c 2}=3.41^{\circ}$ is accompanied by fast growth of both $\mathrm{NM}$ absorption coefficients in the region of prevailing longitudinal absorption, $\hbar \omega \gtrsim 10$ Ry; the peak at $\hbar \omega \simeq 14-15$ Ry becomes $\simeq 40$ times higher when $\theta$ changes from $1^{\circ}$ to $5^{\circ}$. The coefficients also approach each other at these frequencies when $\theta$ approaches $\theta_{c 2}$, and separate again when $\theta$ moves away from $\theta_{c 2}$ (see also Fig. 8). The resonance of right-circular absorption around 5 Ry remains almost unchanged in one mode, and its admixture to the other mode grows $\propto \sin ^{4} \theta$. 
Figure 7 demonstrates behavior of $\mu_{j}(\omega)$ at intermediate $\theta$. The left panel $\left(\theta=30^{\circ}\right)$ shows a typical frequency dependence at $\theta_{c 2}<\theta<\theta_{c 1}$. The NM polarization is almost circular around the critical frequency $\hbar \omega_{c 1}=4.75 \mathrm{Ry}$, and the absorption coefficients can be evaluated there from equations (76) and (77). The main distinction from the case of small $\theta$ is that the right-circular absorption gives a larger contribution to $\mu_{1}$, so that the resonance in that NM mode became much more pronounced. At $\hbar \omega \gtrsim 6 \mathrm{Ry}$ and $\lesssim 4 \mathrm{Ry}$, the NMs are polarized almost linearly, (see Figure 5), and their absorption coefficients are given by equation (75). It should be noted, however, that $\mu_{2}$ in equation (75) corresponds to the solid line at $\hbar \omega \gtrsim 6 \mathrm{Ry}$, and to the dashed line at $\hbar \omega \lesssim 4 \mathrm{Ry}$. The reason is that in the presence of the critical frequencies and non-orthogonality points a unique labeling of the NMs along the continuous curves of the NM absorption and refraction is impossible (see Pavlov et al. 1980); this fact, however, does not impose any difficulties because both NMs participate in the radiative transfer, and their labeling is purely conventional.

The angle $\theta=41^{\circ}$ only slightly exceeds $\theta_{c 1}=40.35^{\circ}$. At this angle the NMs are substantially non-orthogonal near $\omega_{c 1}$, and the absorption coefficients nearly coincide in this region of the right-circular resonance (the middle panel of Fig. 7). At frequencies below and above this region, the NM polarization is close to linear, and the NM behavior is similar to that for $\theta=30^{\circ}$.

At the angle $\theta=75^{\circ}$, which is well above $\theta_{c 1}$, the NM polarizations are almost linear at all frequencies, although there is a very narrow region of enhanced ellipticity at $\hbar \omega \simeq 5.1$ Ry (see Fig. 5). The absorption coefficients are fairly accurately described by equation (75) $\left(\mu_{2}\right.$ corresponds to the solid curve for all the frequencies). Notice that $\mu_{1}$ and $\mu_{2}$ coincide just in the enhanced ellipticity region.

Figure 8 presents the absorption coefficients $\mu_{j}$ as a function of the propagation angle for a few characteristic frequencies. At low frequencies $\omega<\omega_{c 1}$ (see the panel for $\hbar \omega=2$ Ry in Fig. 8), where the absorption coefficients are determined by the magnetically broadened wings of the bound-bound lines, the NM polarization changes from circular to linear with increasing angle. Absorption in one mode slightly decreases with $\theta$, while in the other mode it grows very fast at small angles because of increasing contribution of $\mu_{0}$ (see eq. [77]); the coefficients become close to each other at $\theta \gtrsim 20^{\circ}$, where the NMs are almost linearly polarized.

The picture changes considerably when we approach the first critical frequency, $\hbar \omega_{c 1}=4.751$ Ry. Here, for $\theta=\theta_{c 1}=40.35^{\circ}$, the (linear) polarizations and the absorption coefficients of the modes coincide with each other: $\delta_{1}=\delta_{2}=\pi / 4$ and $\mathcal{P}_{1}=\mathcal{P}_{2}=0$. The modes are linearly polarized at angles above $\theta_{c 1}$, and they become more orthogonal with increasing $\theta$. Below $\theta_{c 1}$, their polarizations are elliptical, and the ellipticity increases with 
decreasing angle.

In the region between the critical frequencies, $\omega_{c 1}<\omega<\omega_{c 2}$, ( $\hbar \omega=7$ Ry in Fig. 8), the longitudinal absorption $\mu_{0}$ (the low-frequency wing of the longitudinal resonance) is much greater than the right-circular absorption $\mu_{+1}$ (the high-frequency wing of the spectral line and the low-frequency wing of the photoionization edge). The NM polarization is nearly circular at small $\theta$ ( $\lesssim 10^{\circ}$ for $\hbar \omega=7 \mathrm{Ry}$ ), and, in accordance with equations (76) and (77), both $\mu_{1}$ and $\mu_{2}$ grow with $\theta: \mu_{1} \simeq \mu_{+1}+\left(\mu_{0} / 2\right) \sin ^{2} \theta, \mu_{2} \simeq\left(\mu_{0} / 2\right) \sin ^{2} \theta$. The polarization becomes almost linear at $\theta \gtrsim 20^{\circ}$, where $\mu_{2} \simeq \mu_{0} \sin ^{2} \theta$ keeps growing, whereas $\mu_{1}=\left(\mu_{+1} / 2\right)+\left(\mu_{0} / \tilde{q}^{2}\right) \cot ^{2} \theta$ decreases because of diminishing contribution from the longitudinal absorption.

At the second critical frequency $\hbar \omega_{c 2}=14.22 \mathrm{Ry}$, the NMs coincide at the critical angle $\theta_{c 2}=3.41^{\circ}$. For $\theta<\theta_{c 2}$, the NMs modes are nearly circular, and the absorption coefficients increase with angle, being very close to each other (cf. Fig. 6) because the both are mainly contributed from the longitudinal absorption $\left(\mu_{0} / \mu_{+1} \simeq 1.3 \times 10^{5}\right.$ at this frequency). For $\theta>\theta_{c 2}$, the linearly polarized NMs become more orthogonal with increasing $\theta: \delta_{2} \rightarrow 0$ and $\delta_{1} \rightarrow \pi / 2$; absorption coefficients of the ordinary and extraordinary modes grow up to $\mu_{0}$ and decrease down to $\mu_{+1} / 2$, respectively.

Behavior of $\mu_{j}(\theta)$ above the second critical frequency is demonstrated in Figure 8 for $\hbar \omega=14.82 \mathrm{Ry}$, near the peak of longitudinal absorption, and for $\hbar \omega=30 \mathrm{Ry}$, where both $\mu_{0}$ and $\mu_{1}$ are determined by the bound-free transitions. At these frequencies, the NM polarizations are virtually orthogonal for any $\theta$. Since $|\tilde{q}| \gg 1$, the polarizations are circular in a narrow range of angles, $\sin \theta \lesssim \tilde{q}^{-1 / 2}$, and they are linear in a wide range of angles. The absorption coefficient of the ordinary mode grows monotonically with $\theta: \mu_{2} \simeq \mu_{+1}+\left(\mu_{0} / 2\right) \sin ^{2} \theta$ at small $\theta$, and $\mu_{2} \simeq \mu_{0} \sin ^{2} \theta$ at larger angles. For the extraordinary mode, $\mu_{1} \simeq\left(\mu_{0} / 2\right) \sin ^{2} \theta$ grows at small $\theta$, reaches its maximum value, $\sim \mu_{0} / \tilde{q}$ at $\sin \theta \sim \tilde{q}^{-1 / 2}$, and then decreases gradually, $\mu_{1} \simeq\left(\mu_{0} / \tilde{q}^{2}\right) \cot ^{2} \theta+\mu_{+1} / 2$.

\section{DISCUSSION}

In this paper we suggested a convenient method for calculating the absorption coefficients of the normal modes in a magnetized medium. Given the 'basic' absorption coefficients $\mu_{0}$ (for the linear polarization along $\mathbf{B}$ at transverse propagation) and $\mu_{ \pm 1}$ (for the circular polarizations at longitudinal propagation), we obtain the antihermitian part of the polarizability tensor and use the Kramers-Kronig transformation to calculate its 
hermitian part. The knowledge of the full polarizability tensor allows us to calculate the polarizations and the absorption coefficients of the NMs for any frequency and direction of propagation. We applied the method to a nonionized, strongly magnetized hydrogen gas for conditions expected in atmospheres of neutron stars. Absorption and propagation of radiation in such a medium is determined by the bound-bound and bound-free transitions. The corresponding spectral lines and photoionization edges are strongly broadened due to distortion of the structure of atoms moving across the magnetic fields, and the shapes of the lines depend essentially on the direction of propagation and polarization of radiation. As a result, the angular and spectral dependences of the NM characteristics are much more complicated than in a fully ionized plasma. In particular, the presence of the nonionized component leads to appearance of additional critical frequencies and angles, where peculiarities of the NM polarizations cause additional crossings of the curves $\mu_{j}(\omega)$ or $\mu_{j}(\theta)$.

The resulting absorption coefficients can be used to solve the radiative transfer equations for the NMs in neutron star atmospheres and to calculate spectra, angular distribution and polarization of emergent radiation. Since the temperature grows inward in the atmosphere, the spectrum and direction of the radiation emitted is mainly determined by the NM which has lower opacity and, therefore, escapes from deeper and hotter layers. We see from Figure 6 that at small $\theta$ the NM of lower opacity shows a strong, asymmetric resonance associated with the bound-bound and bound-free transitions for the longitudinal polarization. Consequently, we should expect a broad, asymmetric absorption feature (at $\hbar \omega \sim 14-15$ Ry in our example) in the emergent spectrum observed at directions close to the magnetic field direction (e. g., when the neutron star is seen pole-on). Figure 7 shows that at intermediate and large $\theta$ the strongest absorption in the NM of lower opacity is that at the bound-bound resonance for the right-circular polarization (at $\sim 5 \mathrm{Ry}$ ), so that the corresponding absorption feature should be most clearly seen at these directions. From the angular dependences of $\mu_{j}$ shown in Figure 8 we see that at most frequencies the NM of lower opacity sharply grows with $\theta$ at small $\theta$, reaches a maximum, and then decreases gradually when $\theta \rightarrow 90^{\circ}$. This means that the angular distribution $I(\theta)$ of the intensity of radiation is beamed along the magnetic field (a 'pencil component' of the angular distribution); a typical width of the beam is $\Delta \theta \lesssim \tilde{q}^{-1 / 2}$ at $\tilde{q} \gg 1\left(\Delta \theta \sim 3^{\circ}-10^{\circ}\right.$ at $\hbar \omega \sim 6-30$ Ry in our example). The maximum of $\mu_{j}(\theta)$ should correspond to a minimum of $I(\theta)$, i. e., a broad 'fan component' in the plane perpendicular to the magnetic field is emitted in addition to the sharp 'pencil component'. The pencil component broadens when the frequency approaches $\omega_{c 1}$; at $\omega<\omega_{c 1}$ the minimum of $I(\theta)$ (and the fan component) disappears. The polarization of the emergent radiation is expected to be strongly polarized at frequencies and angles where the the NM absorption coefficients are substantially 
different. The polarization is circular in the narrow pencil component, and it is linear (perpendicular to the magnetic field) in the fan component.

In the present paper we considered only the case of low temperatures, when the atoms are expected to be nonionized and the effect of their motion on the radiative transitions can be considered in the framework of the perturbation theory. At higher temperatures, the ionized component of the plasma would contribute to the polarization tensor, NM polarizations, and NM opacities. This will result in even more complicated spectral and angular behavior of the NM characteristics. To include both ionized and nonionized components, one should first solve the nontrivial problem of ionization equilibrium of a strongly magnetized, highly nonideal plasma. Including the (nonperturbative) effects of the atomic motion at higher temperatures (Bezchastnov \& Potekhin 1994; Pavlov \& Potekhin 1995) would be also desirable, particularly for propagation at directions close to the magnetic field. Besides, it is possible that not only hydrogen, but also other atoms (e.g. helium, or even iron) can be present in neutron star atmospheres. The study of the structure and radiative transitions in the heavier atoms in very strong magnetic fields is a very complicated problem. When these problems are solved, the results can be easily used to calculate the NM properties with the method suggested in the present paper.

The research has been partially supported through NASA grants NAG5-2807, NAG5-2868 and NASW-4690. We are grateful to Alexander Potekhin who supplied the codes for calculating the photoionization cross section used in this paper. We thank Victor Bezchastnov, Cole Miller, and Yura Shibanov for useful discussions. 


\begin{tabular}{|c|c|c|c|c|c|c|c|}
\hline $\begin{array}{c}\text { Polari- } \\
\text { zation } \\
\alpha\end{array}$ & $\begin{array}{c}\text { Final } \\
\text { level } \\
N^{\prime} s^{\prime} n^{\prime}\end{array}$ & $\begin{array}{c}\text { Final } \\
\text { energy } \\
{[\mathrm{Ry}]}\end{array}$ & $\begin{array}{c}\text { Transition } \\
\text { energy } \\
{[\mathrm{Ry}]}\end{array}$ & $\begin{array}{c}\text { Oscillator } \\
\text { strength } \\
f\end{array}$ & $\begin{array}{l}\hbar \Gamma_{M} \\
{[\mathrm{Ry}]}\end{array}$ & $\begin{array}{l}\hbar \Gamma_{L} \\
{[\mathrm{Ry}]}\end{array}$ & $\begin{array}{l}\hbar \Gamma_{D} \\
{[\mathrm{Ry}]}\end{array}$ \\
\hline 0 & 001 & -0.99 & 14.40 & 0.2272 & 0.987 & 0.0076 & 0.0024 \\
\hline 0 & 003 & -0.25 & 15.08 & 0.0245 & 0.998 & 0.0065 & 0.0026 \\
\hline 0 & 005 & -0.11 & 15.21 & 0.0071 & 0.999 & 0.013 & 0.0026 \\
\hline 0 & $00 \epsilon$ & $>0$ & $>15.32$ & 0.74 & 1.00 & & \\
\hline+1 & 010 & -10.19 & 5.14 & 0.0051 & 0.464 & 0.0014 & 0.0012 \\
\hline+1 & $01 \epsilon^{\prime}$ & $>1.09$ & $>16.41$ & 0.0004 & 1.00 & & \\
\hline-1 & $1-10$ & 1988.72 & 2004.05 & 1.971 & 1.00 & 1.037 & 0.341 \\
\hline-1 & $1-1 \epsilon^{\prime}$ & $>2000.00$ & $>2015.32$ & 0.02 & 1.00 & & \\
\hline
\end{tabular}

Table 1: Bound-bound and bound-free transitions from the ground state, their oscilator strengths, and magnetic, Lorentz, and Doppler widths of the associated lines and photoionization edges at $k T=1 \mathrm{Ry}, \rho=1 \mathrm{~g} \mathrm{~cm}^{-3}$, and $B=2.35 \times 10^{12} \mathrm{G}$. 


\section{References}

Bezchastnov, V. G., \& Potekhin, A. Y. 1994, J. Phys. B, 27, 3349.

Canuto, V. \& Ventura, J. 1977, Fundam. Cosmic Phys., 2, 203.

Davydov, A. S. 1976, Quantum Mechanics, Pergamon Press.

Forster, H., Strupat, W., Rösner, W., Wunner, G., Ruder, H., \& Herold, H. 1984, J. Phys. B, $17,1301$.

Ginzburg, V. L. 1970, The Propagation of Electromagnetic Waves in Plasmas, Pergamon Press.

Gnedin, Yu. N., \& Pavlov, G. G. 1974, Sov. Phys. JETP, 39, 301.

Hasegawa H. \& Howard R. 1961, J. Phys. Chem. Solids, 21, 179.

Herold, H., Ruder, H., \& Wunner, G. 1981, J. Phys. B, 14, 751.

Kaminker, A. D,, Pavlov, G. G., \& Shibanov, Yu. A. 1982, Ap\&SS, 86, 249.

Kaminker, A. D., Pavlov, G. G., \& Shibanov, Yu. A. 1983, Ap\&SS, 91, 167.

Landau, L. D., \& Lifshitz, E. M. 1960, Electrodynamics of Continuous Media, Pergamon Press.

Mészáros, P. 1992, High Energy Radiation from Compact Objects, University of Chicago Press.

Ögelman, H. 1995, in The Lives of the Neutron Stars, eds. A. Alpar, Ü. Kiziloğlu and J. van Paradijs (Dordrecht: Kluwer).

Pavlov, G. G., Shibanov, Yu. A., \& Yakovlev, D. G. 1980, Ap\&SS, 73, 33.

Pavlov, G. G., \& Mészáros, P. 1993, ApJ, 416, 752.

Pavlov, G. G., Shibanov, Yu. A., Zavlin, V. E., \& Meyer, R. D. 1995, in The Lives of the Neutron Stars, eds. A. Alpar, Ü. Kiziloğlu and J. van Paradijs (Dordrecht: Kluwer)

Pavlov, G. G., \& Potekhin, A. Y. 1995, ApJ, 450, 883.

Pavlov, G. G., Stringfellow, G. S., \& Córdova, A. F. 1995, ApJ, submitted.

Potekhin, A. Y. 1994, J. Phys. B, 1994, 27, 1073.

Potekhin, A. and Pavlov, G.: 1993, Ap.J., 407, 330. 
Schmitt, W., Herold, H., Ruder, H., \& Wunner, G, 1981, A\&A, 94, 194.

Sobelman, I, I., Vainshtein, L. A., \& Yukov, E. A. 1981, Excitation of Atoms and Broadening of Spectral Lines, Springer-Verlag.

Vincke, M., \& Baye, D. 1988, J. Phys. B, 212407.

Vincke, M., Le Dourneuf, M., \& Baye, D. 1992, J. Phys. B, 25, 2787. 


\section{Figure Captions}

Figure 1. Frequency dependences of the absorption coefficients $\mu_{\alpha}$ for the three polarizations: $\alpha=0$ (linear polarization along the magnetic field $\mathbf{B}$ ), and $\alpha= \pm 1$ (circular polarizations in the plane perpendicular to $\mathbf{B}$ ). The right panel shows detailed structure of $\mu_{0}$ and $\mu_{+1}$ in the range of bound-bound resonances and bound-free edges. The contributions of the bound-bound (bb) and bound-free (bf) transitions are shown separately. The low-frequency wings of the resonances are determined by the magnetically broadened spectral lines.

Figure 2. The upper panel shows the circular components $\chi_{ \pm 1}$ of the hermitian and antihermitian parts polarizability tensor. The left-circular component $\chi_{-}^{A}$ is very small in this frequency range. The major features of $\chi_{+}^{A}$ and $\chi_{+}^{H}$ are associated with the bound-bound resonance at 5.1 Ry. The lower panel shows $\chi_{0}^{A}$ and $\chi_{0}^{H}$. The main features (shown in the inset separately) are due to the photoionization edge at 15.3 Ry and bound-bound resonance at $14.4 \mathrm{Ry}$.

Figure 3. The upper panel shows the global behavior of the parameters $\tilde{q}$ and $\tilde{p}$ which determine the NM polarization. The two lower panels show $\tilde{q}$ and $\tilde{p}$ in the vicinity of the critical frequencies where $\tilde{q}=0$. Note the different vertical scales.

Figure 4. The solid and dashed curves connect the points in the $\omega-\theta$ plane for which $|q|=1$ and $|p|=1$, respectively. The labels $c_{1}$ and $c_{2}$ denote the non-orthogonality points where $q=0$ and $|p|=1$.

Figure 5. The left three panels show ellipticity and the right three panels show position angles of the NM polarization ellipses as functions of frequency for three angles between $\mathbf{B}$ and direction of propagation: $\theta=1^{\circ}, 30^{\circ}$, and $75^{\circ}$. Note the different scale in the lower panels.

Figure 6. Absorption coefficients of the NMs vs. frequency for three directions of propagation close to $\mathbf{B}$.

Figure 7. Absorption coefficients of the NMs vs. frequency for three intermediate angles $\theta$.

Figure 8. Angular dependences of the absorption coefficients of the NMs for six photon energies. 

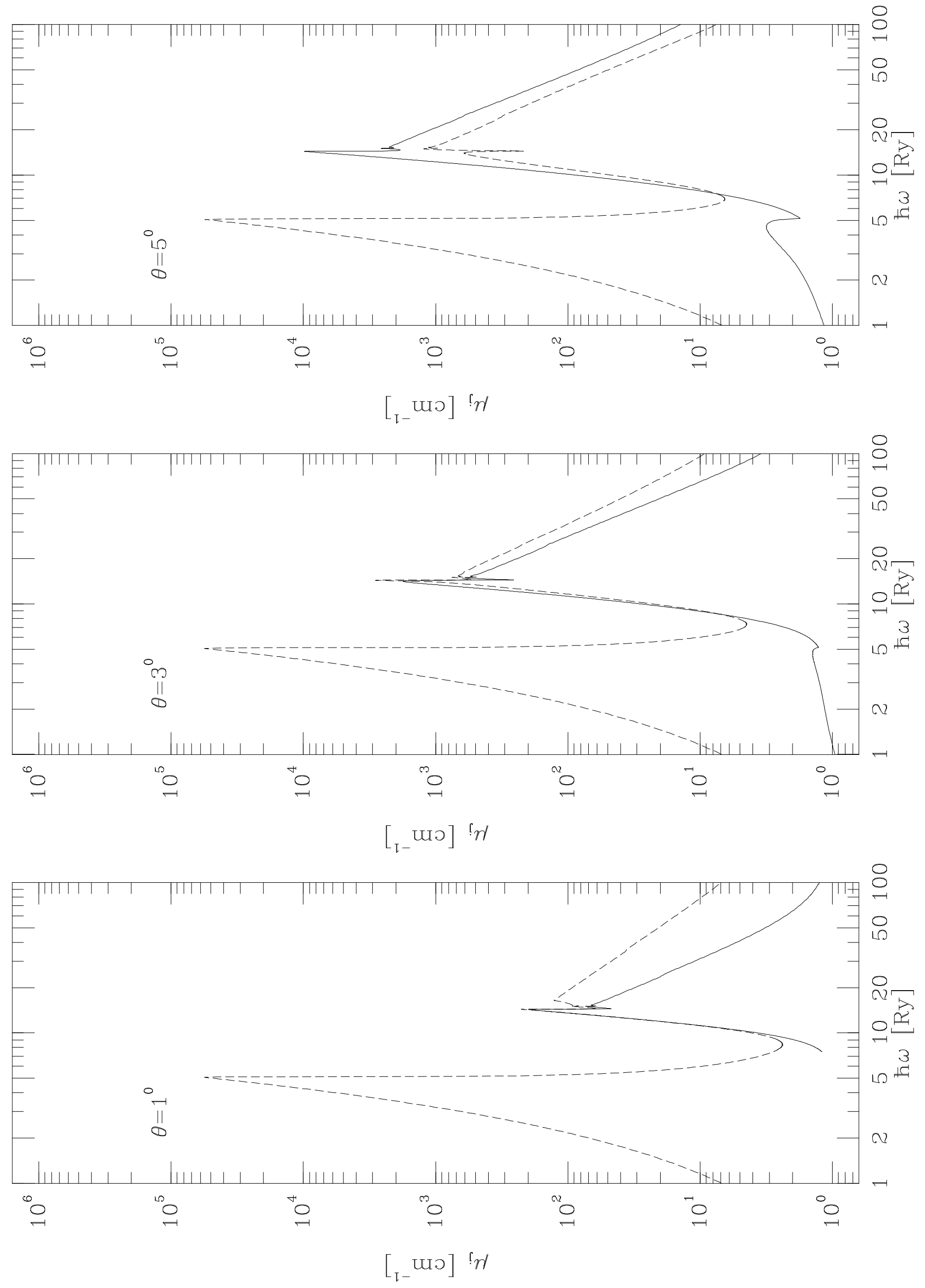

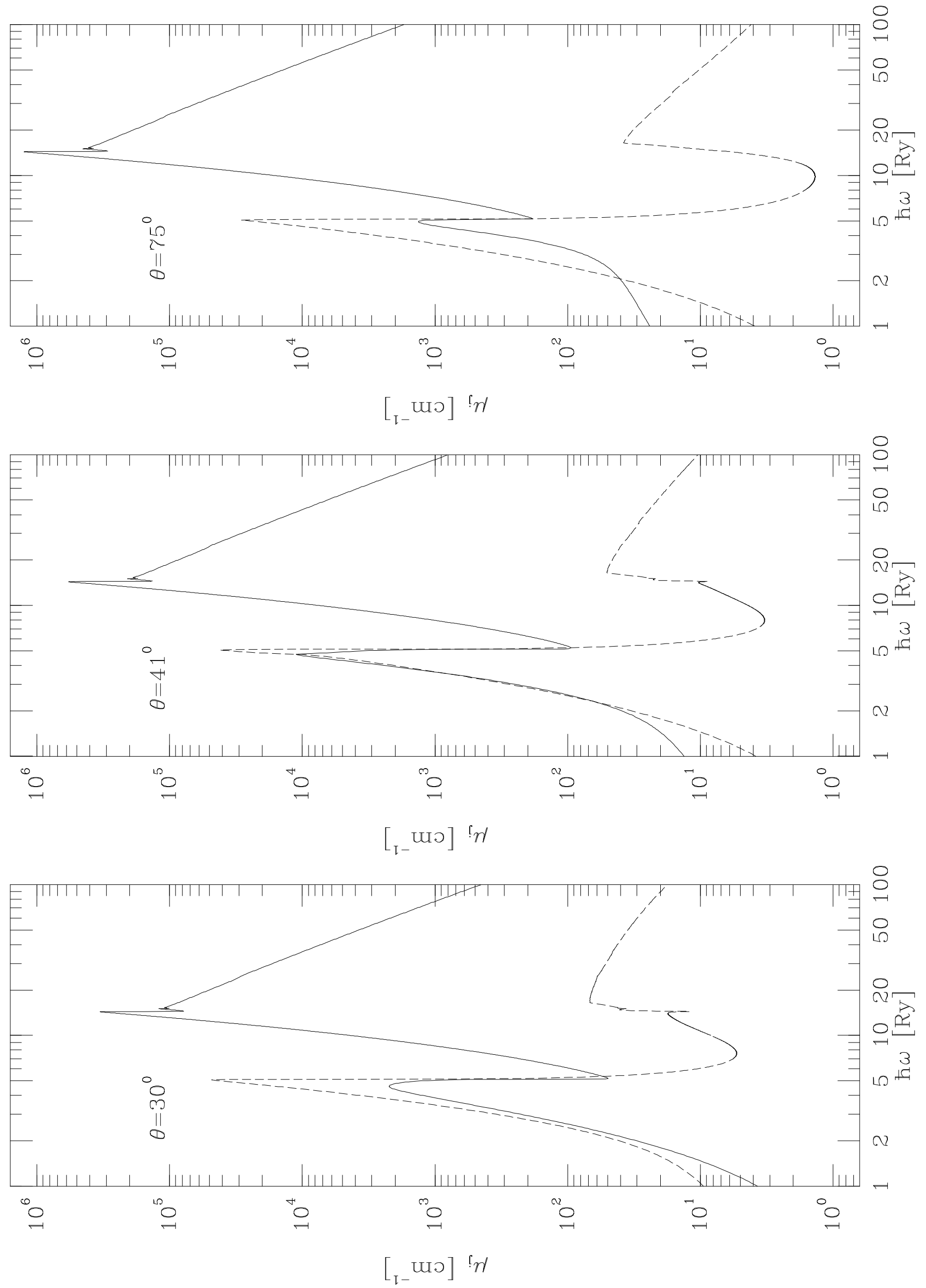

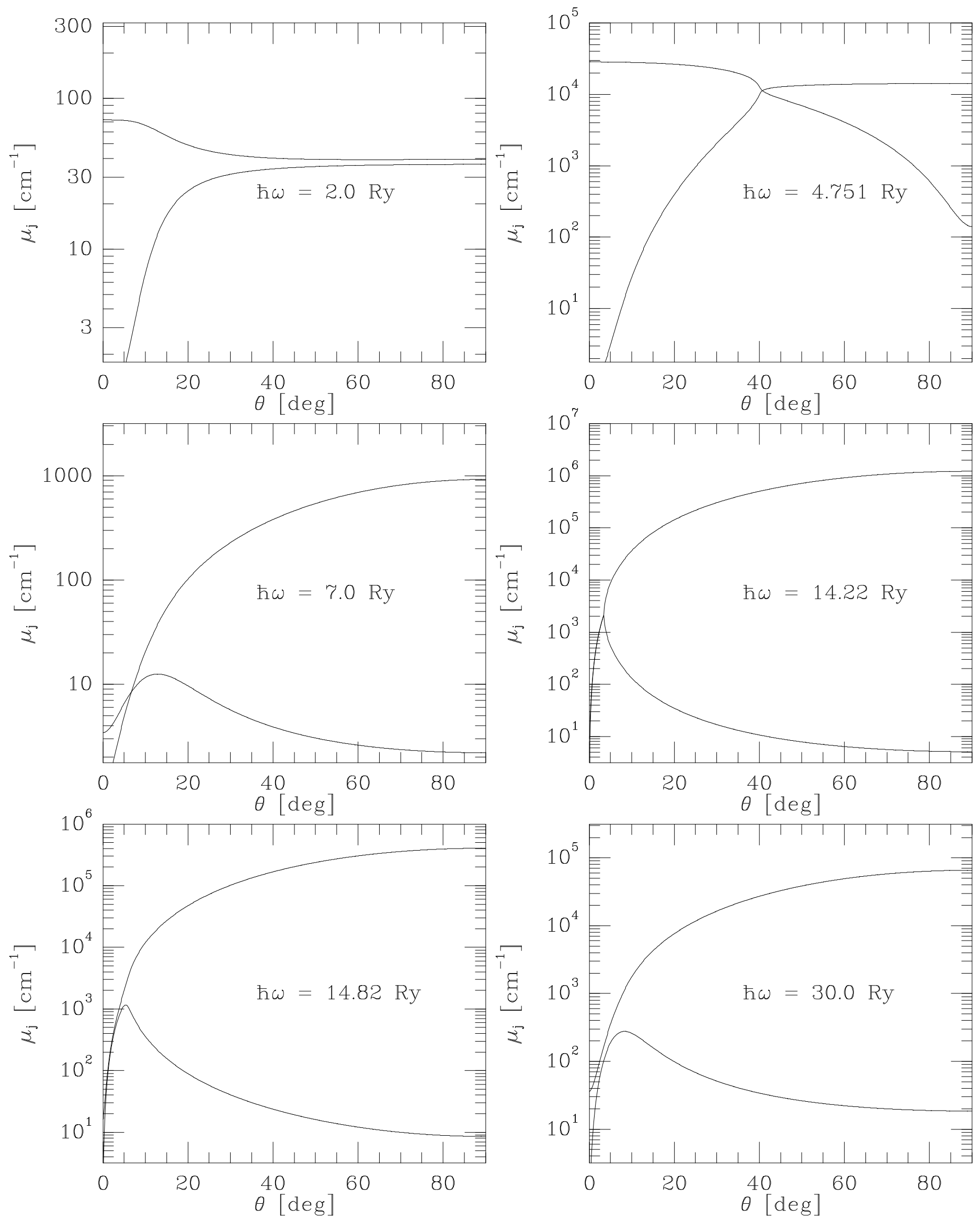

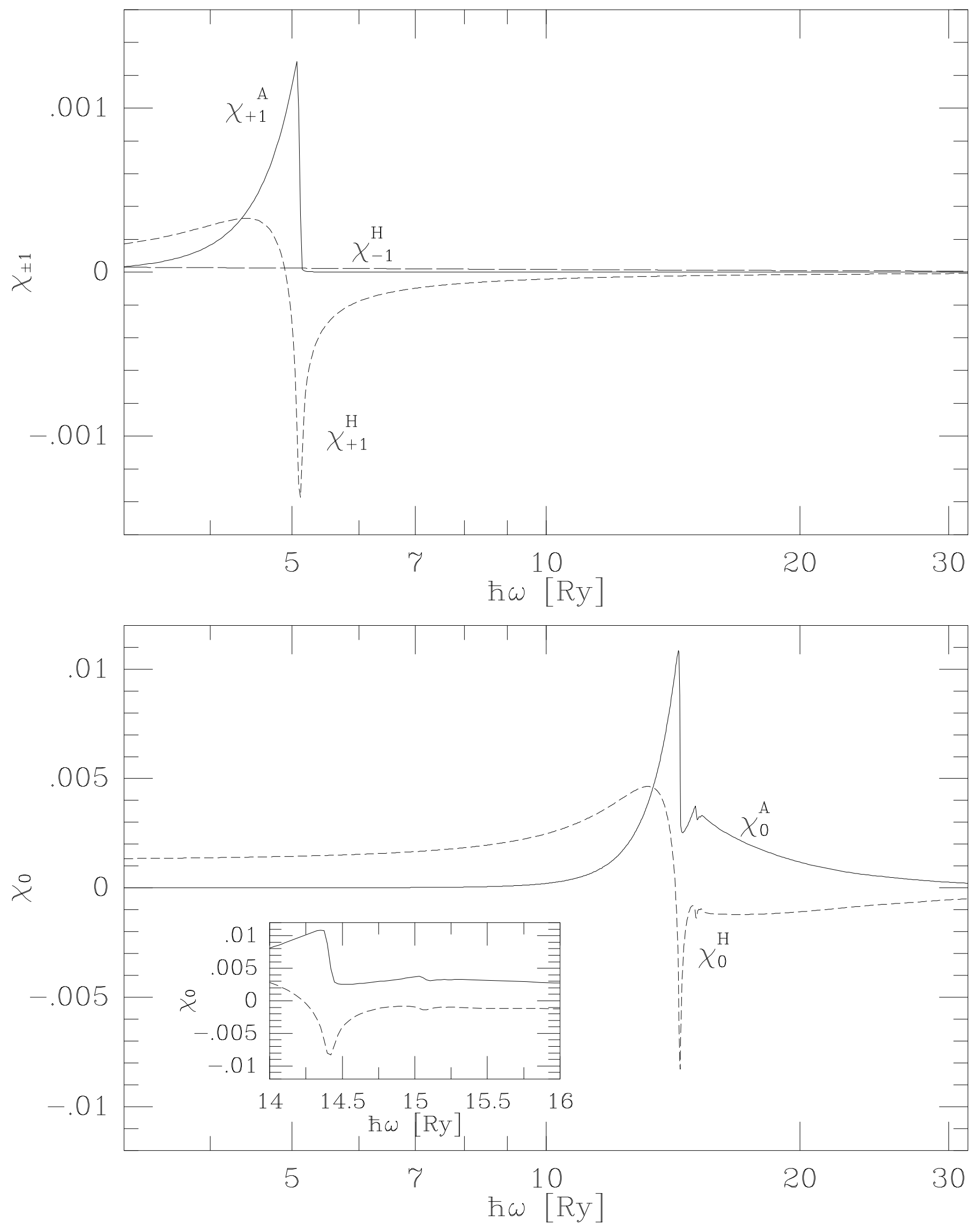

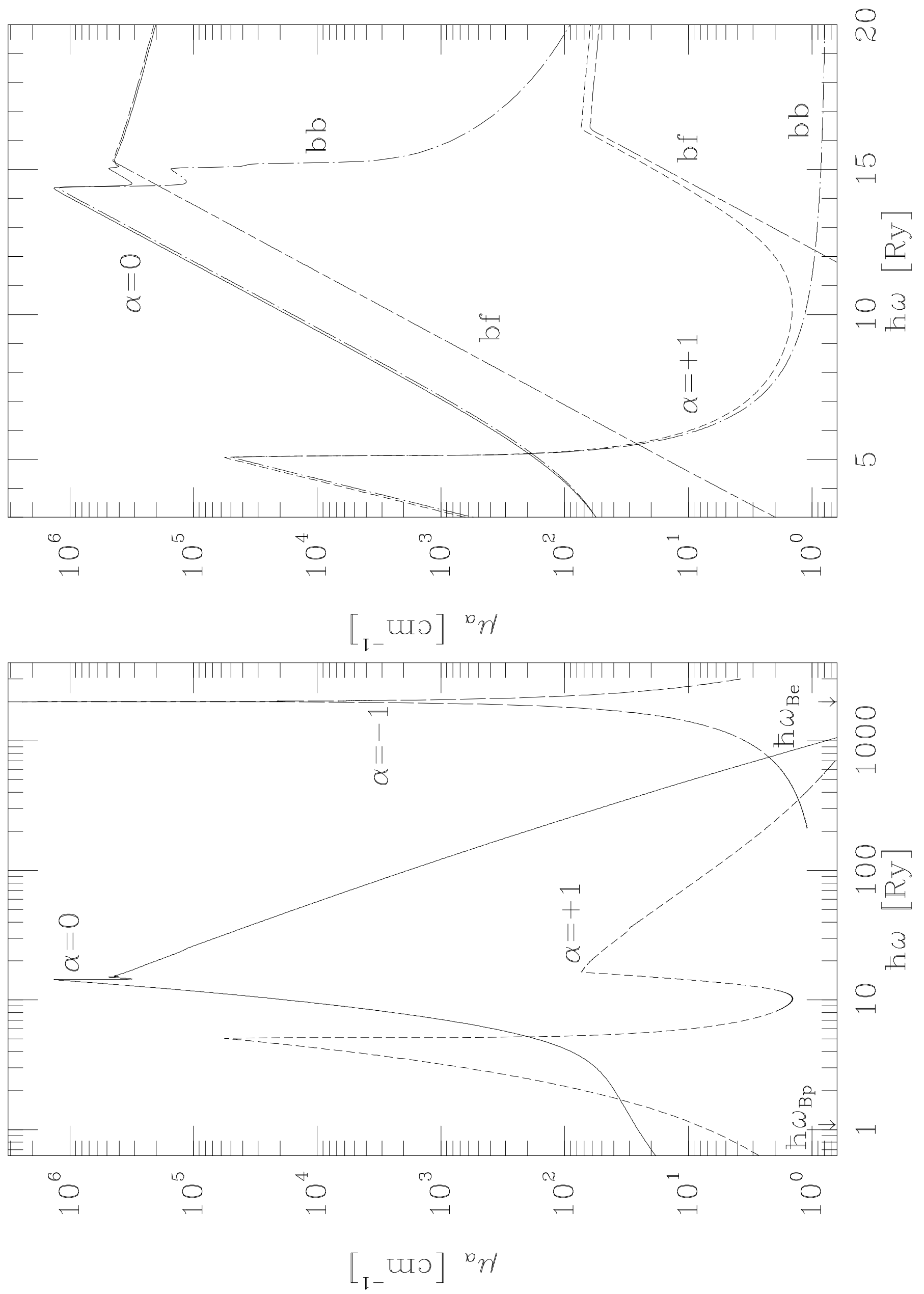

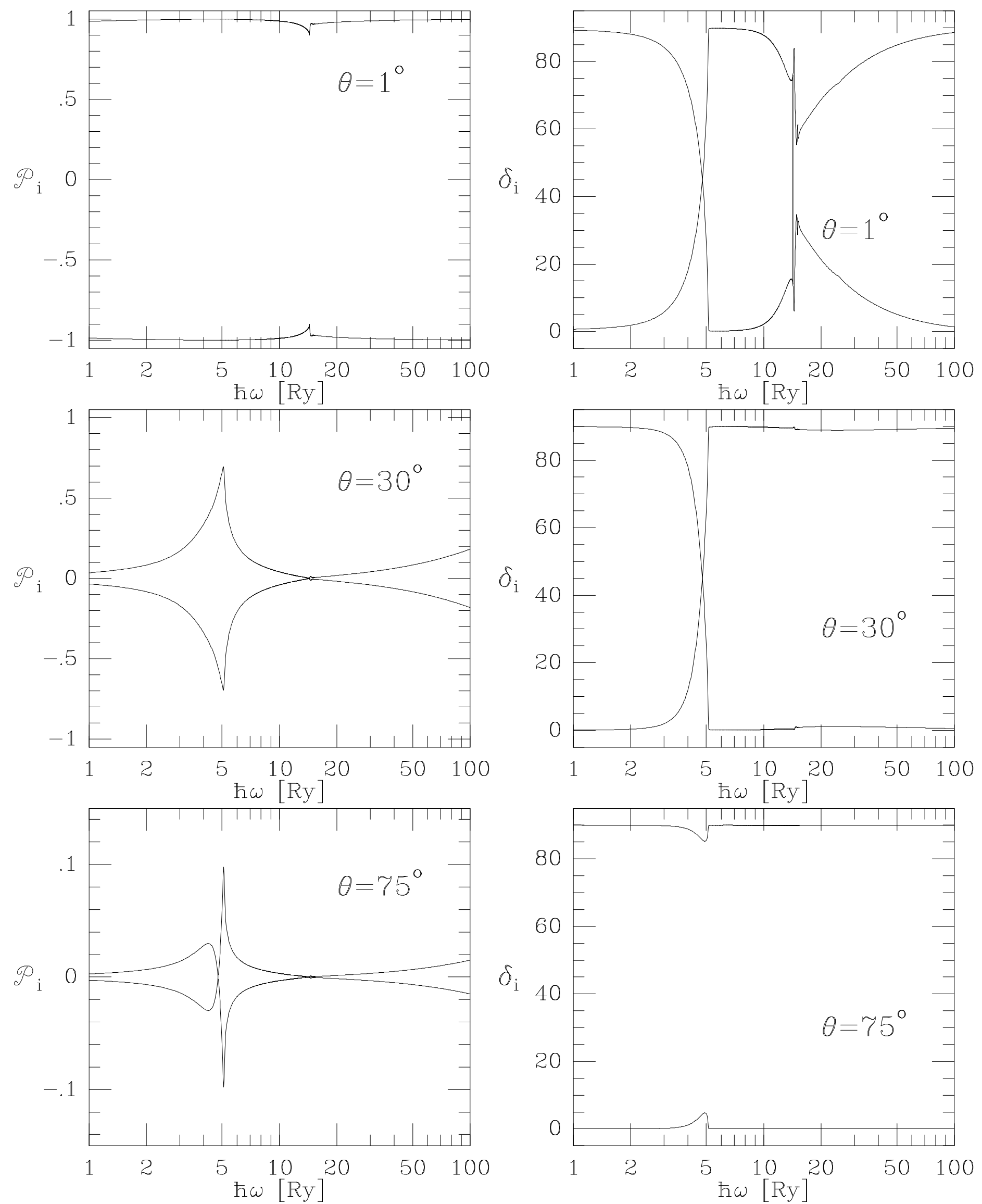

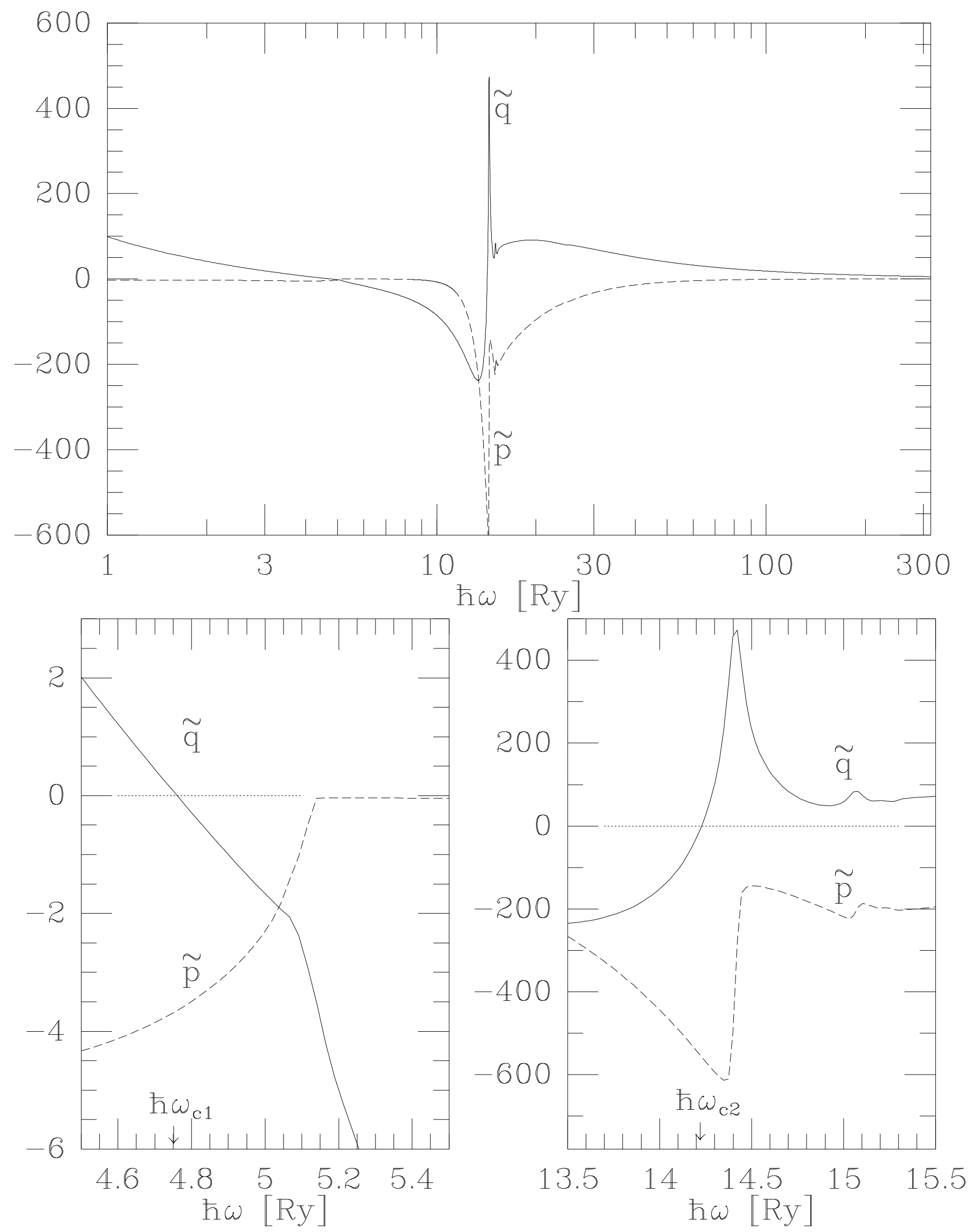


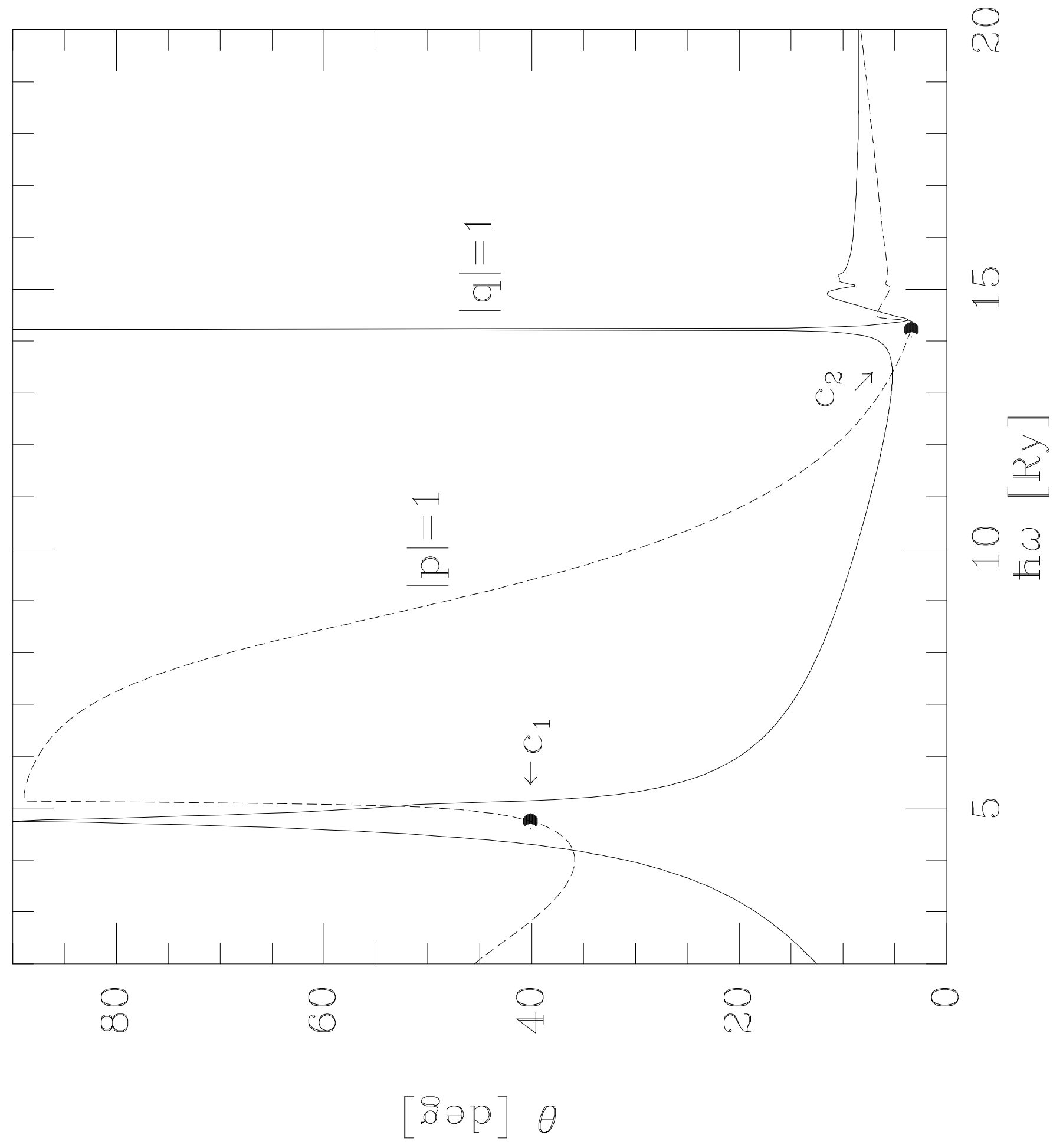

